\title{
Integrability and Huygens' Principle on Symmetric Spaces
}

\author{
Oleg A. Chalykh, Alexander P. Veselov ${ }^{\star}$ \\ Department of Mathematics and Mechanics, Moscow State University, 119899 Moscow, Russia
}

Received: 9 June 1995/Accepted: 17 July 1995

\begin{abstract}
The explicit formulas for fundamental solutions of the modified wave equations on certain symmetric spaces are found. These symmetric spaces have the following characteristic property: all multiplicities of their restricted roots are even. As a corollary in the odd-dimensional case one has that the Huygens' principle in Hadamard's sense for these equations is fulfilled. We consider also the heat and Laplace equations on such a symmetric space and give explicitly the corresponding fundamental solutions-heat kernel and Green's function. This continues our previous investigations [16] of the spherical functions on the same symmetric spaces based on the fact that the radial part of the Laplace-Beltrami operator on such a space is related to the algebraically integrable case of the generalised CalogeroSutherland-Moser quantum system. In the last section of this paper we apply the methods of Heckman and Opdam to extend our results to some other symmetric spaces, in particular to complex and quaternian grassmannians.
\end{abstract}

\section{Introduction}

It is well-known that the behaviour of the solutions of the wave equation on the plane and in the space is rather different. A pointwise disturbance in $\mathbf{R}^{3}$ generates a pure spherical wave, while on the plane the disturbed domain is the whole disc. In the first case we say that Huygens' principle holds (in Hadamard's sense), in the latter we have the wave diffusion (see e.g. [1]).

More precisely, Huygens' principle (HP) for a second order hyperbolic equation means that the fundamental solution of the corresponding Cauchy problem is located on the characteristic conoid. The problem of finding all hyperbolic equations with HP is known as Hadamard's problem. In his fundamental lectures on the Cauchy problem [2] in Yale University in 1923 Hadamard found the criterion for the validity of HP and as a result he proved that HP is impossible in the even-dimensional

$\star$ Present address: Department of Mathematical Sciences, Loughborough University of Technology, Loughborough, Leicestershire, LE11 3TU, United Kingdom

e-mail address: A.P.Veselov@lut.ac.uk 
case. Unfortunately, this criterion turned out to be not effective enough to solve Hadamard's problem, which is open until now.

At first it was believed that all equations with HP are equivalent to the usual wave equation. But in 1953 Stellmacher found an example of a non-wave huygensian equation in $\mathbf{R}^{5}$, and then in 1965 Günther found the equations with HP corresponding to non-trivial metrics in four-dimensional space-time. The reader can find more details of the history of HP and Hadamard's problem in Günther's book [3].

New examples of the equations with HP in the flat space have been found recently by $\mathrm{Yu}$. Berest and one of the authors $[4,5]$. The starting point for these investigations was the comparison of the important papers $[6,7]$ by Stellmacher and Lagnese devoted to the so-called restricted Hadamard's problem with the results of the modern theory of the finite-gap Schrödinger operators in one dimension (see e.g. [8]). In many dimensions there is no such complete theory; there are some partial results only (mainly in dimension 2) [9-13]. In particular in [12] it was suggested that the Schrödinger operators given by the quantum hamiltonians of the Calogero-Sutherland-Moser (CSM) problem and its generalisations found by Olshanetsky and Perelomov [15] with the special values of parameters are "finitegap" or algebraic, and moreover under some assumptions they exhaust all such operators. This conjecture was later partially proved (see $[16,17]$ ).

This was the motivation to consider these operators in the context of Hadamard's problem in [4] and in the present paper. On the intuitive level it is natural because these operators are "reflection-less" in a sense and this property is very close to HP. But we should mention that as follows from the results of Lagnese and Stellmacher $[6,7]$ the wave equation with the general soliton (Bargmann) potential does not satisfy HP; it holds for the corresponding rational limits only. The same is true for the generalised CSM problem (see [5]).

It turns out that the trigonometric versions of the generalised CSM problem nevertheless in some special cases are related to Huygens' principle not in the euclidean but in certain symmetric spaces. The corresponding symmetric spaces are rather special: all their root multiplicities have to be even.

Here is the list of the irreducible globally symmetric spaces with even root multiplicities.

\section{List 1}

Compact type

1. Odd-dimensional spheres $\mathbf{S}^{2 n+1}$.

2. Simple compact Lie groups.

3. Symmetric spaces of AII type in Cartan's notations

$$
\mathbf{X}=\mathbf{S U}(2 n) / \mathbf{S p}(n) .
$$

4. Exceptional 26-dimensional symmetric space of EIV type $\mathbf{X}=\mathbf{E}_{6} / \mathbf{F}_{4}$.

Noncompact type

1. Odd-dimensional Lobachevsky or hyperbolic spaces $\mathbf{H}^{2 n+1}$.

2. Symmetric spaces with a complex group of motions: $\mathbf{X}=\mathbf{G} / \mathbf{K}, \mathbf{G}$ is a simple complex Lie group considered as a real one, $\mathbf{K}$ is its maximal compact subgroup. 
3. Symmetric spaces of AII type:

$$
\mathbf{X}=\mathbf{S U}^{*}(2 n) / \mathbf{S p}(n), \quad \operatorname{dim} \mathbf{X}=(n-1)(2 n+1) .
$$

Here $\mathbf{S U}^{*}(2 n)$ is a quaternian analogue of $\mathbf{S L}(n)$ :

$$
\mathbf{S U}^{*}(2 n)=\mathbf{S L}(2 n, \mathbf{C}) \cap \mathbf{G L}(n, \mathbf{H})
$$

and $\mathbf{S p}(n)$ is its intersection with $\mathbf{U}(2 n)$ (see [18]).

4. Exceptional symmetric space of EIV type which is dual to the space $\mathbf{X}=\mathbf{E}_{6} / \mathbf{F}_{4}$ and related to Cayley numbers or octonions (see $[18,19]$ ).

These spaces in the noncompact case have been discussed in our previous paper [16] (see also [20]), where we have found some explicit formulas for the spherical functions and the inversion of the integral Abel's transform on the spaces of AII type based on the fact that the radial parts of the corresponding LaplaceBeltrami operators are conjugate to the algebraically integrable cases of the trigonometric version of the CSM problem. It is interesting to note that the same symmetric spaces are characterized by the property that their horocycle Radon transform can be inverted by means of a differential operator [21,61].

Exactly the same spaces appeared to be considered already in the theory of Huygens' principle. Namely, consider the wave equation on symmetric space $\mathbf{X}$ :

$$
\varphi_{t t}-\mathscr{L}_{X} \varphi=0,
$$

where $\mathscr{L}_{X}$ is the Laplace-Beltrami operator on $\mathbf{X}$. If $\mathbf{X}$ is flat and odd-dimensional then this equation satisfies HP in accordance with classical results by Tedone [1]. On a sphere however HP for Eq. (1) never holds (see e.g. [22]), while the modified wave equation

$$
\varphi_{t t}-\mathscr{L}_{X} \varphi+c \varphi=0
$$

satisfies HP for a suitable constant $c$. For the unit odd-dimensional sphere $\mathbf{S}^{n}$ the corresponding constant is

$$
c=\left(\frac{n-1}{2}\right)^{2}
$$

(see P. Günther [23] for $n=3$ and Lax-Phillips [24] for all odd $n$ ).

For an arbitrary irreducible symmetric space $\mathbf{X}$ the constant $c$ has the form

$$
c= \pm|\rho|^{2}, \quad \rho=\frac{1}{2} \sum_{\alpha \in \mathscr{R}_{+}} m_{\alpha} \alpha .
$$

Here $\mathscr{R}_{+}$is a set of positive restricted roots of $\mathbf{X}, \rho$ is half of their sum with the multiplicities and \pm is the sign of the curvature of $\mathbf{X}$ : plus for spaces of compact type and minus for spaces of noncompact type (see e.g. [18]). For a reducible space $c$ is the sum of the constants corresponding to its irreducible components.

Note that Eq. (2) as well as (1) is invariant under the group of isometries of $\mathbf{X}$. spaces.

There is the following result about the modified wave equation on symmetric

Theorem. If all root multiplicities $m_{\alpha}$ of an odd-dimensional symmetric space $\mathbf{X}$ are even then the modified wave equation (2),(3) on $\mathbf{X}$ satisfies Huygens' principle.

For symmetric spaces of noncompact type this result belongs to Solomatina [25], the detailed proof of it was given by Olafsson, Schlichtkrull [26] and Helgason [27]. The case of symmetric spaces with a complex group of motion was considered 
earlier by Helgason $[29,28]$. We should mention also the paper by Semenov-TianShansky [30] where HP is established on the same symmetric spaces but for a certain system of equations with many "times."

For the modified wave equation on a compact semisimple Lie group HP was established by Helgason [28, 29] (see also the paper by Dowker [31]). As it concerns the general case of compact symmetric spaces with even root multiplicities we have not found the proof of the corresponding result in the literature although the specialists seem to have no doubt about it after the papers [25-27].

In the present paper we give the explicit formulas for the fundamental solution of the Cauchy problem for the modified wave equation (2) on the symmetric spaces listed above. From these formulas Huygens' principle for these equations and the formulated theorem follow immediately. In the forthcoming paper [32] we use the results about the generalised Calogero-Moser problem to prove the inverse statement, i.e. that for modified wave equations Huygens' principle holds only for symmetric spaces with even multiplicities.

We consider also the Laplace equation $\mathscr{L}_{X} \phi=0$ as well its modified version

$$
\left(\mathscr{L}_{X}-c\right) \phi=0
$$

and heat equation

$$
u_{t}=\mathscr{L}_{X} u
$$

on such a symmetric space and give the corresponding fundamental solutions - the heat kernel and Green's function-explicitly. In various special cases such formulas were known before, see e.g. [23,28,31,33-37,39] (it should be noted that the formulas for the heat kernels on compact simple Lie groups in paper [38] seems to be wrong).

Notice that the form of the heat kernel essentially depends on the global properties of the corresponding manifold in contrast to Huygens' principle, which is a local phenomenon. In particular, for the symmetric spaces of compact type the heat kernels are expressed via theta-functions while for noncompact type-1pt in elementary functions.

In the last section we use the shift operator of Heckman and Opdam [44,45] to find some less explicit formulas for the fundamental solutions of the modified wave, Laplace and heat equations on certain other symmetric spaces.

More precisely, these spaces are characterized by the following properties: 1) their root system $\mathscr{R}$ is of $\mathbf{B C}_{n}$ type, 2) if we identify $\mathscr{R}$ with the set $\left\{ \pm e_{i}, \pm 2 e_{i}\right.$, $\left.\pm e_{i} \pm e_{j}\right\}$ in $\mathbf{R}^{n}$ then the roots $\pm e_{i} \pm e_{j}$ have even multiplicity (for the spaces of rank 1 this is supposed to be fulfilled automatically).

Here is the list of all such irreducible globally symmetric spaces $\mathbf{X}$ and the corresponding values $k=\left(k_{1}, k_{2}, k_{3}\right)$, where $2 k_{1}, 2 k_{2}$ and $2 k_{3}$ are the multiplicities $m_{\alpha}$ of the roots $\alpha= \pm e_{i}, \pm 2 e_{i}$ and $\pm e_{i} \pm e_{j}(i<j)$. For the spaces of rank 1 one has only $k_{1}$ and $k_{2}$. Notice that Lists 1 and 2 cover all symmetric spaces of rank 1 .

\section{List 2}

1. Even-dimensional spheres $\mathbf{S}^{2 n}$ and Lobachevsky spaces $\mathbf{H}^{2 n}$, the rank is 1 , $k=(0, n-1 / 2)$. 
2. Complex grassmannians and their duals, type AIII in Cartan's notation:

$\mathbf{S U}(p, q) / \mathbf{S}\left(\mathbf{U}_{p} \times \mathbf{U}_{q}\right)$ and $\mathbf{S U}(p+q) / \mathbf{S}\left(\mathbf{U}_{p} \times \mathbf{U}_{q}\right)(p \leqq q)$, the rank equals $p$ and $k=(q-p, 1 / 2,1)$ if $p>1$. When $p=1$, i.e. for complex projective spaces $\mathbf{C P}^{\mathbf{q}}$ and their dual hyperbolic spaces $k=(q-1,1 / 2)$.

3. Quaternian grassmannians and their duals, type CII: $\mathbf{S p}(p, q) / \mathbf{S p}(p) \times \mathbf{S p}(q)$ and $\mathbf{S p}(p+q) / \mathbf{S p}(p) \times \mathbf{S p}(q)(p \leqq q)$, the rank equals $p$ and $k=(2 q-2 p, 3 / 2,2)$ if $p>1$. When $p=1$, i.e. for the quaternian projective spaces $\mathbf{H P}^{\mathbf{q}}$ and their dual hyperbolic spaces $k=(2 q-2,3 / 2)$.

4. The spaces of complex structures in $\mathbf{R}^{2 n}$ and their duals, type DIII: $\mathbf{S O}^{*}(2 n) /$ $\mathbf{U}(n)$ and $\mathbf{S O}(2 n) / \mathbf{U}(n)$, the rank is the entire $\frac{n}{2}$ and $k=(1 / 2,0,2)$ for even $n$ and $k=(2,1 / 2,2)$ for odd $n$.

5. Exceptional spaces of type EIII (of rank 2) and EVII (of rank 3 ) with the following $k:(4,1 / 2,3)$ for EIII and $(0,1 / 2,4)$ for EVII.

6. Projective Cayley plane $\mathbf{C a P}^{2}$ and its dual hyperbolic space, type FII in Cartan's list, the rank is $1, k=(4,7 / 2)$.

By using the shift operator we reduce the problem of finding the fundamental solutions for the modified wave, Laplace and heat equations on these spaces to that for the spaces, which are much simpler: for the complex grassmannians with odd $p+q$ they are the products of the complex projective planes, for the rest the products of two-dimensional spheres (or hyperbolic planes in the noncompact case). As far as we know the first observation of such a kind belongs to Berezin and Karpelevich [53].

\section{Radial Parts of Laplace-Beltrami Operators and Shift Operators}

We restrict ourselves to the noncompact type since all formulas for the compact type can be obtained from the formulas below by changing hyperbolic functions to their trigonometric counterparts.

So let $\mathbf{X}=\mathbf{G} / \mathbf{K}$ be a symmetric space of the noncompact type, $\mathbf{G}$ be a real semisimple group with finite center, $\mathbf{K}$ be its maximal compact subgroup. Consider the corresponding Cartan's decomposition [18] $\mathbf{G}=\mathbf{K A K}$. Let $\mathscr{G}$ and $\mathscr{A}$ be Lie algebras of groups $\mathbf{G}$ and $\mathbf{A}$ and $\mathscr{R}$ denotes the set of the roots of $\mathscr{G}$ with respect to $\mathscr{A}, \mathscr{R} \subset \mathscr{A}^{*}$. It is known that the roots $\alpha$ may have non-unit multiplicities $m_{\alpha}$ in contrast to the theory of complex simple Lie algebras. We write $\mathscr{R}_{+}$for the set of positive roots and $\rho$ for half of their sum with multiplicities:

$$
\rho=\frac{1}{2} \sum_{\alpha \in \mathscr{R}} m_{\alpha} \alpha .
$$

Introduce the "polar" coordinates $(r, k)$ on $\mathbf{X}$ in accordance with Cartan's decomposition

$$
\mathbf{G}=\mathbf{K A K}: g \mathbf{K}=k \exp r \mathbf{K},
$$

where $g \mathbf{K}=\mathbf{G} / \mathbf{K}=\mathbf{X}, r \in \mathscr{A}, k \in \mathbf{K}$. It should be noted that the coordinates ("radii") $r=\left(r_{1}, \ldots, r_{n}\right), n=\operatorname{dim} \mathscr{A}=\mathrm{rk} \mathbf{X}$, are defined up to the action of the Weyl group $\mathbf{W}$. The action of the Laplacian $\mathscr{L}_{X}$ on the "radial" functions is equivalent to that of the following operator:

$$
\mathscr{L}_{X}^{\mathrm{rad}}=\Delta+\sum_{\alpha \in \mathscr{R}_{+}} m_{\alpha} \operatorname{coth}(\alpha, r)\left(\alpha, \frac{\partial}{\partial r}\right),
$$


where $\Delta$ is the usual Laplacian in $\mathbf{R}^{n} \cong \mathscr{A}$ [43]. The operator $\mathscr{L}_{X}^{\text {rad }}$ is called the radial part of the Laplace-Beltrami operator $\mathscr{L}_{X}$.

Theorem 1.1. If all root multiplicities $m_{\alpha}$ are even, then there exists a differential operator $\mathscr{D}\left(r, \frac{\partial}{\partial r}\right)$ with the following property:

$$
\mathscr{L}_{X}^{\mathrm{rad}} \circ \mathscr{D}=\mathscr{D} \circ\left(\Delta-|\rho|^{2}\right) .
$$

For the rank one spaces it follows from [35, 36, 23]. For the spaces $\mathbf{X}=\mathbf{G} / \mathbf{K}$ with complex $\mathbf{G}$, i.e. for the case when all $m_{\alpha}=2$, this result is essentially equivalent to the observation made by F. Berezin [41]. For the spaces of AII type $\left(m_{\alpha}=4\right)$ of rank 2 and 3 as well as for the space of EIV type $\left(m_{\alpha}=8\right)$ the explicit formulas for the operator $\mathscr{D}$ can be extracted from $[47,48]$ for all spaces of AII type-from $[12,16,20]$.

E. Opdam [44] established the existence of such an operator called the shift operator for some formal generalizations of the radial parts of the LaplaceBeltrami operators with arbitary even multiplicities $m_{\alpha}$. Later G. Heckman [45] gave an effective construction of the shift operators; we will describe it at the end of this section.

The operator $\mathscr{D}\left(r, \frac{\partial}{\partial r}\right)$ has a form

$$
\mathscr{D}=\prod_{\alpha \in \mathscr{R}_{+}}(\sinh (\alpha, r))^{-\frac{1}{2} m_{\alpha}} \prod_{\alpha \in \mathscr{R}_{+}} \partial_{\alpha}^{\frac{1}{2} m_{\alpha}}+\cdots,
$$

where $\partial_{\alpha}=\left(\alpha, \frac{\partial}{\partial r}\right)$ and the dots mean the lower terms. The operator of this form is completely determined by the property (7) if its coefficients are the rational functions of $\exp (\alpha, r), \alpha \in \mathscr{R}_{+}$. This implies that $\mathscr{D}$ is invariant under the Weyl group.

Now we give the explicit formulas for the operators $\mathscr{D}$ for all irreducible symmetric spaces with even root multiplicities (see List 1). For such a reducible space the shift operator is a product of the operators corresponding to the irreducible components.

1. For the hyperbolic space $\mathbf{X}=\mathbf{H}^{2 n+1}$ we have only one radius $r=r_{1}$ and

$$
\mathscr{L}_{H^{2 n+1}}^{\text {rad }}=\frac{\partial^{2}}{\partial r^{2}}+2 n \operatorname{coth} r \frac{\partial}{\partial r} .
$$

It is easy to check that

$$
\mathscr{D}=\left(\frac{1}{\sinh r} \frac{\partial}{\partial r}\right)^{n}
$$

satisfies the relation

$$
\left(\frac{\partial^{2}}{\partial r^{2}}+2 n \operatorname{coth} r \frac{\partial}{\partial r}\right) \circ \mathscr{D}=\mathscr{D} \circ\left(\frac{\partial^{2}}{\partial r^{2}}-n^{2}\right),
$$

and is therefore the shift operator (compare with $[35,36]$ ).

2 . For the symmetric spaces $\mathbf{X}=\mathbf{G} / \mathbf{K}$ with a complex group $\mathbf{G}$ all root multiplicities $m_{\alpha}=2$ and the operator $\mathscr{D}$ has the form (compare with [41]):

$$
\mathscr{D}=\prod_{\alpha \in \mathscr{R}_{+}} \sinh ^{-1}(\alpha, r) \prod_{\alpha \in \mathscr{R}_{+}} \partial_{\alpha}
$$


3. For the symmetric spaces of AII type, $\mathbf{X}=\mathbf{S U}^{*}(2 m) / \mathbf{S p}(m)$, the Cartan subalgebra consists of all real diagonal matrices diag $\left(r_{1}, \ldots, r_{m}, r_{1}, \ldots, r_{m}\right)$ with $r_{1}+\cdots+r_{m}=0$. The roots have the form $\alpha=r_{i}-r_{j}$, and all $m_{\alpha}=4$. We normalize the invariant metric on $\mathbf{G}$ by the condition that its restriction to $\mathscr{A}$ is the standard euclidean structure.

Introduce the following operator:

$$
\mathscr{D}=\prod_{i<j} \sinh ^{-2}\left(r_{i}-r_{j}\right) \mathscr{D}_{m} \circ \mathscr{D}_{m-1} \circ \cdots \circ \mathscr{D}_{2} \circ \prod_{i<j}\left(\frac{\partial}{\partial r_{i}}-\frac{\partial}{\partial r_{j}}\right),
$$

where the differential operators $\mathscr{D}_{l}(l=2, \ldots, n)$ are determined as a result of replacing $z_{i}$ by $\frac{\partial}{\partial r_{i}}-\frac{\partial}{\partial r_{l}}$ in the following polynomial on $z$ with the coefficients depending on $r$ :

$$
\mathscr{D}_{l}(r, z)=\exp \left(-\sum_{i<l} 2 \operatorname{coth}\left(r_{i}-r_{l}\right) \frac{\partial}{\partial z_{i}}+\sum_{i<j<l} 2 \sinh ^{-2}\left(r_{i}-r_{j}\right) \frac{\partial^{2}}{\partial z_{i} \partial z_{j}}\right) \prod_{i<l} z_{i} .
$$

The operator $\mathscr{D}$ defined in this way is the shift operator in that case (see [16]).

4. For the space of EIV type the shift operator has the form [47, 48, 42]:

$$
\mathscr{D}=\left(\sinh r_{12} \sinh r_{23} \sinh r_{31}\right)^{-4} \mathscr{D}_{3} \circ \mathscr{D}_{2} \circ \mathscr{D}_{1} \circ \mathscr{D}_{0},
$$

where

$$
\begin{aligned}
\mathscr{D}_{l}= & \mathrm{D}_{12} \circ \mathrm{D}_{23} \circ \mathrm{D}_{31}-2 l f_{12} \mathrm{D}_{23} \circ \mathrm{D}_{31}-2 l f_{23} \mathrm{D}_{31} \circ \mathrm{D}_{12}-2 l f_{31} \mathrm{D}_{12} \circ \mathrm{D}_{23} \\
& +\left(4 l^{2} f_{12} f_{23}-l(l-1) f_{31}^{2}\right) \mathrm{D}_{31}+\left(4 l^{2} f_{23} f_{31}-l(l-1) f_{12}^{2}\right) \mathrm{D}_{12} \\
& +\left(4 l^{2} f_{31} f_{12}-l(l-1) f_{23}^{2}\right) \mathrm{D}_{23}-2 l(l-1)(l+2)\left(f_{12}^{3}+f_{23}^{3}+f_{31}^{3}\right) \\
& -6 l^{2}(l+1) f_{12} f_{23} f_{31}+4 l(2 l-1)\left(f_{12}+f_{23}+f_{31}\right), \\
\mathrm{D}_{i j}= & \frac{\partial}{\partial r_{i}}-\frac{\partial}{\partial r_{j}}, \quad f_{i j}=\operatorname{coth} r_{i j}, \quad r_{i j}=r_{i}-r_{j}, \quad r_{1}+r_{2}+r_{3}=0 .
\end{aligned}
$$

At the end of this section we describe the general procedure proposed by Heckman [45] to construct the shift operators for an arbitrary root system.

Consider any root system $\mathscr{R} \subset \mathscr{A}^{*}$ and an arbitary $\mathbf{W}$-invariant function $k(\alpha)=k_{\alpha}$ on $\mathscr{R}$. For a fixed set of positive roots $\mathscr{R}_{+} \subset \mathscr{R}$ and $\xi \in \mathscr{A}$ we define the following difference-differential operator $\nabla_{\xi}$ on the space of functions of $f(r), r \in A$ which is the trigonometric version of the Dunkl operator [46]:

$$
\nabla_{\xi}=\left(\xi, \frac{\partial}{\partial r}\right)+\sum_{d \in R_{+}} k_{\alpha}(\xi, \alpha) \operatorname{coth}(\alpha, r)\left(1-\hat{s}_{\alpha}\right),
$$

where $\left(\xi, \frac{\partial}{\partial r}\right)$ is the derivative in the direction $\xi:\left(\xi, \frac{\partial}{\partial r}\right)=\sum_{i=1}^{n} \xi^{i} \frac{\partial}{\partial r^{i}}$ in $\mathbf{R}^{n} \cong \mathscr{A}$, and the operator $\hat{s}_{\alpha}$ acts on a function $f$ of $r$ in the following way:

$$
\left(\hat{s}_{\alpha} f\right)(r)=f\left(s_{\alpha}(r)\right),
$$

$s_{\alpha}$ is the orthogonal reflection in $\mathscr{A}$ corresponding to the root $\alpha: s_{\alpha}(r)=r-2 \frac{(\alpha, r)}{(\alpha, \alpha)} \alpha$ (we identify $\mathscr{A}$ and $\mathscr{A}^{*}$ with the help of Killing's form and suppose that $\mathscr{R} \subset \mathscr{A}$ ). It will be important for us in the next section that the operator $\nabla_{\xi}$ is regular. 
Suppose now that the system $\mathscr{R}$ is reduced (the case $\mathscr{R}=B C_{n}$ we discuss in the last section) and let $S \subset \mathscr{R}$ be one of the orbits of Weyl group $\mathbf{W}$ in $\mathscr{R}$. The orbit $S$ defines the character of the group $\mathbf{W} \varepsilon_{S}: \mathbf{W} \rightarrow \pm 1$ in the following way: $\varepsilon_{S}\left(s_{\alpha}\right)=-1$ for $\alpha \in S$ and $\varepsilon_{S}\left(s_{\alpha}\right)=+1$ for other $\alpha \in \mathscr{R}$. Put $S_{+}=S \cap \mathscr{R}_{+}$ and

$$
\pi_{S}(r)=\prod_{\alpha \in S_{+}} \sinh (\alpha, r)
$$

We write $d$ for the number of elements in $S_{+}$and define for any $\xi \in \mathscr{A}$ the following differential operator $D_{k, S}$ :

$$
D_{k, S}=\operatorname{Res}\left(\pi_{S}^{-1} \sum_{w \in \mathbf{W}} \varepsilon_{S}(w) \nabla_{w \xi}^{d}\right),
$$

where $\nabla_{w \xi}$ is defined by (13), and Res denotes taking the differential part by means of the restriction of the operator onto the space $\mathscr{F} \mathbf{W}$ of $\mathbf{W}$-invariant functions. For an algebraic combination of the operators (13) such a procedure is welldefined. Indeed, all operators $\hat{s}_{\alpha}$ can be transposed to the right using the commutation laws:

$$
\begin{aligned}
\hat{s}_{\alpha} \circ\left(\xi, \frac{\partial}{\partial r}\right) & =\left(s_{\alpha} \xi, \frac{\partial}{\partial r}\right) \circ \hat{s}_{\alpha}, \\
\hat{s}_{\alpha} \circ f & =\left(\hat{s}_{\alpha} f\right) \circ \hat{s}_{\alpha},
\end{aligned}
$$

and then one should replace all $\hat{s}_{\alpha}$ by the identity operator. Remark that a differential operator on $\mathscr{A}$ is determined completely by its restriction to the space $\mathscr{F} \mathbf{W}$.

Let's introduce the following differential operator $\mathscr{L}_{k}$ on $\mathscr{A}$ :

$$
\mathscr{L}_{k}=\Delta+2 \sum_{\alpha \in \mathscr{R}_{+}} k_{\alpha} \operatorname{coth}(\alpha, r)\left(\alpha, \frac{\partial}{\partial r}\right)+|\rho(k)|^{2}
$$

where $k=\left(k_{\alpha}\right)_{\alpha \in \mathscr{R}}$ is any $\mathbf{W}$-invariant function, $\rho(k)=\sum_{\alpha \in \mathscr{R}_{+}} k_{\alpha} \alpha$, and $\Delta$ is the Laplacian in $\mathbf{R}^{n} \cong \mathscr{A}$.

Theorem 1.2 [45]. For the operators (14) and (15) the following relation holds:

$$
D_{k, S} \circ \mathscr{L}_{k}=\mathscr{L}_{k+1_{S}} \circ D_{k, S}
$$

where $1_{S}$ is the characteristic function of the set $S$, which is equal to 1 on $S$ and zero on the other roots.

The relation (16) means that the operator $D_{k, S}$ transforms the eigenfunctions of $\mathscr{L}_{k}$ to the eigenfunctions of $\mathscr{L}_{k+1_{S}}$ and shifts in that sense from $k$ to $k+1_{S}$. Now Theorem 1.1 follows immediately from this result because for $k_{\alpha}=\frac{1}{2} m_{\alpha}$ one has $\mathscr{L}_{k}=\mathscr{L}_{\mathbf{X}}^{\text {rad }}+|\rho|^{2}$, while for $k \equiv 0 \quad \mathscr{L}_{0}=\Delta$. Furthermore, the highest symbol of the operator (14) has the form $\pi_{S}^{-1} \sum_{w \in \mathbf{W}} \varepsilon_{w}\left(w \xi, \frac{\partial}{\partial r}\right)^{d}$ and up to a coefficient is $\pi_{S}^{-1}(r) \pi_{S}\left(\frac{\partial}{\partial r}\right)$. So one can obtain the shift operator $\mathscr{D}$ from Theorem 1.1 as the composition of the "elementary" shift operators (14) each of them increasing for 2 the multiplicities $m_{\alpha}$ of the roots $\alpha \in S$. 


\section{Fundamental Solution of the Modified Wave Equation on Symmetric Space}

Let $\mathbf{X}$ be an odd-dimensional symmetric space with even root multiplicities of noncompact type. Now we find the fundamental solution $\Phi(x, \xi), x, \xi \in \mathbf{X}$ of the corresponding Cauchy problem for the modified wave equation on $\mathbf{X}$ :

$$
\Phi_{t t}-\left(\mathscr{L}_{X}+|\rho|^{2}\right) \Phi=0,\left.\quad \Phi\right|_{t=0}=0,\left.\quad \Phi_{t}\right|_{t=0}=\delta_{\xi}(x),
$$

where $\delta_{\xi}(x)$ is the delta-function located at the point $\xi$.

Remark that it is sufficient to find the solution $F(x, t)$ of the equation

$$
F_{t t}-\left(\mathscr{L}_{X}+|\rho|^{2}\right) F=0
$$

with the initial data

$$
\left.F\right|_{t=0}=0,\left.\quad F_{t}\right|_{t=0}=\delta(x),
$$

where $\delta(x)$ is the delta function on $\mathbf{X}$, concentrated in the "origin" $x=e \mathbf{K}, e$ is the unit of $\mathbf{G}$. Indeed, one can obtain the fundamental solution

$$
\Phi(g x, g \xi, t)=\Phi(g, \xi, t), \quad g \in \mathbf{G}
$$

for any $\xi=h \mathbf{K}$ from the function $F(x, t)=\Phi(x, e \mathbf{K}, t)$ in the following way:

$$
\Phi(x, \xi, t)=F\left(h^{-1} x, t\right),
$$

because $\mathbf{X}$ is homogeneous and the Laplace-Beltrami operator is invariant. In its turn if one knows $\Phi(x, \xi)$ the fundamental solution $E(x, \xi, t, \tau)$ of the nonhomogeneous modified wave equation

$$
E_{t t}-\left(\mathscr{L}_{X}+|\rho|^{2}\right) E=\delta_{\xi}(x) \delta(t-\tau)
$$

can be found as $E(x, \xi, t, \tau)=\Phi(x, \xi, t)$ if $t>\tau$ and zero otherwise (see e.g. [40], p. 204).

Introduce the following distribution on $\mathbf{X}$ depending on $t$ :

$$
\begin{aligned}
F(x, t) & =C \mathscr{D}\left(r, \frac{\partial}{\partial r}\right) \delta^{\left(\frac{n-3}{2}\right)}\left(t^{2}-|r|^{2}\right), \\
C & =(-1)^{\frac{N-n}{2}} 2^{\frac{n-N-2}{2}} \pi^{\frac{1-N}{2}} .
\end{aligned}
$$

Here $r \in \mathscr{A} \cong \mathbf{R}^{n}$ is the radial component of $x=g \mathbf{K} \in \mathbf{X}$, determined from Cartan's decomposition

$$
g \mathbf{K}=k(\exp r) \mathbf{K},
$$

$N$ and $n$ are the dimension and the rank of $\mathbf{X}$ correspondingly, which for the spaces considered are odd numbers with the relation

$$
N=n+\sum_{\alpha \in \mathscr{R}_{+}} m_{\alpha},
$$

$\delta^{(p)}(\Gamma)$ is the $p^{\text {th }}$ derivative of the delta function $\delta(\Gamma)$ (see [40]), and $\mathscr{D}\left(r, \frac{\partial}{\partial r}\right)$ is the shift operator given by the explicit formulas (9)-(12) in the section before. The coordinates $r=\left(r_{1}, \ldots, r_{n}\right)$ are defined only modulo the action of the Weyl group $\mathbf{W}$ of the system $\mathscr{R}$ (see [18]). But since the operator $\mathscr{D}$ is also $\mathbf{W}$-invariant, 
the formula (22) gives the well-defined distribution on $\mathbf{X}$ which is located on the geodesic sphere with the radius $t$ and with the center in $x=e \mathbf{K}$.

Theorem 2.1. For an odd-dimensional symmetric space $\mathbf{X}$ of the noncompact type with even root multiplicities, the distribution (22) satisfies the modified wave equation (2)-(3) with the initial data (19) and defines its fundamental solution by formula (20).

The proof of the theorem follows from the properties of the operator $\mathscr{D}$ and from $\mathbf{K}$-invariance of $F$. Indeed, note that

$$
\left(\mathscr{L}_{X}+|\rho|^{2}\right) F=\left(\mathscr{L}_{X}^{\mathrm{rad}}+|\rho|^{2}\right) F,
$$

since $F$ is "radial." Then according to (7),

$$
\left(\mathscr{L}_{X}^{\mathrm{rad}}+|\rho|^{2}\right) \circ \mathscr{D}=\mathscr{D} \circ \Delta,
$$

and now while $\delta^{\left(\frac{n-3}{2}\right)}\left(t^{2}-r^{2}\right)$ satisfies the usual wave equation in $\mathbf{R}^{n, 1} F(x, t)$ is the solution of the equation

$$
F_{t t}-\left(\mathscr{L}_{X}+|\rho|^{2}\right) F=0 .
$$

It turns out that due to the properties of the operator $\mathscr{D}, F$ has also the required initial data: $\left.F\right|_{t=0}=0,\left.F_{t}\right|_{t=0}=\delta(x)$. To prove this we recall that the operator $\mathscr{D}$ in formula (22) can be obtained as the composition of the operators (14). In its turn the action of these operators on $\delta^{\left(\frac{n-3}{2}\right)}\left(t^{2}-|r|^{2}\right)$ in formula (22) is equivalent to the action of the corresponding combination of the operators (13) from formula (14). Indeed, the function $\delta^{\left(\frac{n-3}{2}\right)}\left(t^{2}-|r|^{2}\right)$ is $\mathbf{W}$-invariant and hence the procedure of restriction Res is not necessary in that case. Now consider the expression under Res in (14): $\pi_{S}^{-1} \sum_{w \in \mathbf{W}} \varepsilon_{S}(w)\left(\nabla_{w \xi}\right)^{d}$. Note that the operator $\nabla_{\xi}$ is regular and the expression $R=\sum_{w \in \mathbf{W}} \varepsilon_{S}(w)\left(\nabla_{w \xi}\right)^{d}$ is clearly $S$-antisymmetric (i.e. $\left.w R=\varepsilon_{S}(w) R\right)$. Hence the latter expression being divided on $\pi_{S}$ remains regular, that is the operator $\pi_{S}^{-1} \sum_{w \in \mathbf{W}} \varepsilon_{s}(w) \nabla_{w \xi}^{d}$ is also regular. On the other hand acting by $\mathscr{D}$ on $\delta^{\left(\frac{n-3}{2}\right)}\left(t^{2}-|r|^{2}\right)$ we obtain according to (8) the following result:

$$
a_{0}(r) \delta^{\left(\frac{N-3}{2}\right)}\left(t^{2}-|r|^{2}\right)+a_{1}(r) \delta^{\left(\frac{N-5}{2}\right)}\left(t^{2}-|r|^{2}\right)+\cdots+a_{\frac{N-n}{2}}(r) \delta^{\left(\frac{n-3}{2}\right)}\left(t^{2}-|r|^{2}\right)
$$

where $N=\operatorname{dim} X$. Therefore the coefficients $a_{i}(r)$ are regular. It is easy to see from (8) that $a_{0}$ has the form

$$
a_{0}(r)=\prod_{\alpha \in R_{+}}\left(-2(\alpha, r)^{-1} \sinh (\alpha, r)\right)^{-\frac{1}{2} m_{\alpha}} \rightarrow \prod_{\alpha \in R_{+}}(-2)^{-\frac{1}{2} m_{\alpha}},
$$

while $r \rightarrow 0$. As well as in $N$-dimensional euclidean space we have:

$$
\begin{aligned}
\left.\delta^{(s)}\left(t^{2}-|r|^{2}\right)\right|_{t=0} & =0 \text { for } s \leqq \frac{N-3}{2} \\
\left.\frac{\partial}{\partial t}\left(\delta^{(s)}\left(t^{2}-|r|^{2}\right)\right)\right|_{t=0} & = \begin{cases}2 \pi^{\frac{N-1}{2}} \delta(x) & \text { for } s=\frac{N-3}{2} \\
0 & \text { for } s<\frac{N-3}{2} .\end{cases}
\end{aligned}
$$

That gives the required initial data for $F$. 
Remark. For the dual spaces of compact type the fundamental solution for small time can be obtained from the formulas above by replacing the hyperbolic functions by their trigonometric counterparts.

Corollary 2.1. The modified wave equation on an odd-dimensional symmetric space with even root multiplicities satisfies Huygens' principle.

Now we apply the general formula (22) to the spaces from List 1.

For the Lobachevsky space $\mathbf{H}^{2 m+1}$ its rank $n$ equals $1, \delta^{\left(\frac{n-3}{2}\right)}=\delta^{(-1)}$ is the Heaviside function $\theta$ and the formulas (9), (20), (22) give the well-known result

$$
\begin{aligned}
\Phi(x, \xi, t) & =\frac{(-1)^{m}}{2^{m+1} \pi^{m}}\left(\frac{1}{\sinh r} \frac{\mathrm{d}}{\mathrm{d} r}\right)^{m} \theta\left(t^{2}-r^{2}\right) \\
& =\frac{(-1)^{m}}{(2 \pi)^{m}}\left(\frac{1}{\sinh r} \frac{\mathrm{d}}{\mathrm{d} r}\right)^{m-1} \frac{r}{\sinh r} \delta\left(t^{2}-|r|^{2}\right),
\end{aligned}
$$

where $r$ denotes the distance between $x$ and $\xi$.

For a symmetric space $\mathbf{X}=\mathbf{G} / \mathbf{K}$ with a complex group of motions $\mathbf{G}$ of odd complex dimension $N$ all root multiplicities $m_{\alpha}$ are equal to 2 and we obtain:

$$
\begin{aligned}
F(x, t) & =C \prod_{\alpha \in \mathscr{R}_{+}} \sinh ^{-1}(\alpha, r) \prod_{\alpha \in \mathscr{R}_{+}} \partial_{\alpha} \delta^{\left(\frac{n-3}{2}\right)}\left(t^{2}-|r|^{2}\right) \\
& =C(-2)^{p} \prod_{\alpha \in \mathscr{R}_{+}} \frac{(\alpha, r)}{\sinh (\alpha, r)} \delta^{\left(\frac{N-3}{2}\right)}\left(t^{2}-|r|^{2}\right),
\end{aligned}
$$

where $p$ denotes the number of positive roots of $\mathbf{G}$.

The latter equality follows from the following identity, which can be proved by using simple symmetry arguments:

$$
\left(\prod_{\alpha \in \mathscr{R}_{+}} \partial_{\alpha}\right) \delta^{(s)}\left(t^{2}-|r|^{2}\right)=(-2)^{p} \prod_{\alpha \in \mathscr{R}_{+}}(\alpha, r) \delta^{(s+p)}\left(t^{2}-|r|^{2}\right) .
$$

Formula (26) is equivalent to the formula found by Helgason in [28].

Example. The symmetric space $\mathbf{X}=\mathbf{S L}(n, \mathbf{C}) / \mathbf{S U}(n)$ can be identified with the space of the unimodular positive hermitian matrices in accordance with the wellknown polar decomposition of any $g \in \mathbf{S L}(n, \mathbf{C})$ :

$$
g=h u
$$

where the matrices $h$ and $u$ are correspondingly positive hermitian and unitary with determinant 1 . The coordinates $r_{1}, \ldots, r_{n}$ are determined as the logarithms of the eigenvalues of $h$ :

$$
r_{i}=\log \lambda_{i}, r_{1}+\cdots+r_{n}=\log \lambda_{1} \cdots \lambda_{n}=\log \operatorname{det} h=0 .
$$

The corresponding function (22) has the form:

$$
F(h, t)=\frac{1}{2 \pi^{\frac{n^{2}-1}{2}}} \prod_{i<j} \frac{\left(r_{i}-r_{j}\right)}{\sinh \left(r_{i}-r_{j}\right)} \delta^{\left(\frac{n^{2}-4}{2}\right)}\left(t^{2}-|r|^{2}\right) .
$$


For odd-dimensional spaces of AII type we have in accordance with (22),

$$
F(x, t)=C(-2)^{n(2 n-1)}\left(\prod_{i<j} \sinh ^{-2}\left(r_{i}-r_{j}\right)\right) \mathscr{D}\left(\prod_{i<j}\left(r_{i}-r_{j}\right) \delta^{\left(2 n^{2}-2\right)}\left(t^{2}-|r|^{2}\right)\right),
$$

with

$$
\mathscr{D}=\mathscr{D}_{2 n} \circ \mathscr{D}_{2 n-1} \circ \cdots \circ \mathscr{D}_{2},
$$

where the operators $\mathscr{D}_{2}, \ldots, \mathscr{D}_{2 n}$ are given by (20).

Remark 1. For the even-dimensional spaces with even root multiplicities $m_{\alpha}$ (e.g. for the exceptional space EIV) the fundamental solution of the modified wave equation can be obtained in the same way. To do this one should replace $\delta^{\left(\frac{n-3}{2}\right)}\left(t^{2}-\right.$ $\left.|r|^{2}\right)$ in (22) by $\left(\frac{\theta\left(t^{2}-|r|^{2}\right)}{\sqrt{t^{2}-|r|^{2}}}\right)^{\left(\frac{n-2}{2}\right)}$. In this case the fundamental solution is not located on the characteristic conoid so Huygens' principle doesn't hold in agreement with the general Hadamard's result. Remark that for the products of such a space with the analogous odd-dimensional one, e.g. for $\mathbf{X} \times \mathbf{S}^{3}$, Huygens' principle does hold and the fundamental solution is given by Theorem 2.1 .

Remark 2. The modified wave operators $\mathscr{L}=\partial_{t}^{2}-\mathscr{L}_{X} \pm|\rho|^{2}$ for the spaces under consideration admit the wave family

$$
\varphi=\mathscr{L}^{\frac{n-1}{2}} \mathscr{D} f(t-r)
$$

with an arbitrary function $f$, where $\mathscr{D}$ is the shift operator and $n$ is the rank of the symmetric space. This follows from the definition of $\mathscr{D}$ and from Beltrami's identity (see $[1,49])$.

Remark 3. In the papers [35,36] Kiprijanov and Ivanov have considered the so-called Euler-Poisson-Darboux equation on the hyperbolic space $\mathbf{H}^{2 n+1}$ :

$$
\frac{\partial^{2} \varphi}{\partial t^{2}}-\mathscr{L} \varphi-\left(n^{2}+\frac{\lambda}{\sinh ^{2} t}\right) \varphi=0 .
$$

In the special case $(\lambda=0)$ one has the modified wave equation. For a certain singular Cauchy problem and the special values of $\lambda: \lambda=(p-1)(p-3) / 4, p=$ $3,4, \ldots, 2 n+1$ they proved that Huygens' principle holds. The method of the "shift of the parameter" they used goes back to paper [51] and is very similar in its idea to the methods we use.

\section{Laplace Equation on Symmetric Space and its Modified Version}

Let $\mathbf{X}$ be any globally symmetric space of noncompact type, $\mathscr{L}_{X}$ be its LaplaceBeltrami operator. By the Laplace equation on $\mathbf{X}$ we mean the equation

$$
\mathscr{L}_{X} \phi=0 \text {. }
$$

Let's define also the modified Laplace equation on $\mathbf{X}$ as the following elliptic equation:

$$
\left(\mathscr{L}_{X}+|\rho|^{2}\right) \phi=0,
$$


where $\rho$ is the same as above, see (3). The corresponding operator

$$
\hat{\mathscr{L}}_{X}=\mathscr{L}_{X}+|\rho|^{2}
$$

we will call as modified Laplace-Beltrami operator on $\mathbf{X}$. For the general symmetric space it has a form

$$
\hat{\mathscr{L}}_{X}=\mathscr{L}_{X}-c
$$

where $c$ is defined in (3).

The operator $-\mathscr{L}_{X}$ has $\lambda=|\rho|^{2}$ as the bottom of its spectrum; this explains why it is natural to consider the modified equations.

We start from the even more general equation of the form

$$
\left(\mathscr{L}_{X}+\gamma|\rho|^{2}\right) \phi=0
$$

where $\gamma$ is an arbitrary constant. When $\gamma=0$ one has the Laplace equation, when $\gamma=1-$ its modified version.

We are interested in the fundamental solution for this equation, which is a solution of

$$
\left(\mathscr{L}_{X}+\gamma|\rho|^{2}\right) G^{\gamma}(x, \xi)=\delta_{\xi}(x)
$$

where $\delta_{\xi}(x)$ is the delta-function at the point $\xi \in \mathbf{X}$. If one knows it one can find the solution of the corresponding Poisson equation

$$
\left(\mathscr{L}_{X}+\gamma|\rho|^{2}\right) \psi(x)=f(x)
$$

by the formula

$$
\psi(x)=\int G^{\gamma}(x, \xi) f(\xi) d \xi .
$$

To find $G^{\gamma}(x, \xi)$ we use the same method as in the previous section. Let's introduce the radial coordinates $r(x, \xi)$ of the point $x=g \mathbf{K} \in \mathbf{X}$ with respect to the point $\xi=h \mathbf{K}$ by the following Cartan decomposition:

$$
h^{-1} g \mathbf{K}=k(\exp r) \mathbf{K}
$$

(compare with (6)). It is easy to see that $r(x, \xi)=-r(\xi, x)$.

Let's look for a solution $G^{\gamma}(x, \xi)$ depending only on $r(x, \xi)$. In this case we have

$$
\left(\mathscr{L}_{X}+\gamma|\rho|^{2}\right) G^{\gamma}=\left(\mathscr{L}_{X}^{\mathrm{rad}}+\gamma|\rho|^{2}\right) G^{\gamma}
$$

and using the shift operator $\mathscr{D}(7)$ we come to the corresponding euclidean operator:

$$
\left(\mathscr{L}_{X}^{\mathrm{rad}}+\gamma|\rho|^{2}\right) \mathscr{D}=\mathscr{D}\left(\Delta+(\gamma-1)|\rho|^{2}\right) .
$$

Let $G_{0}^{\gamma}$ be the fundamental solution of the operator $\left(\Delta+(\gamma-1)|\rho|^{2}\right)$. Up to some constant it has a form (see e.g. [1]):

$$
G_{0}^{\gamma}=|r|^{\mu} J_{-\mu}(\alpha|r|),
$$

where $\mu=(n-2) / 2, \alpha=\sqrt{(\gamma-1)}|\rho|, J_{\mu}$ is the Bessel function, if $n=\operatorname{rank} \mathbf{X}$ is odd and $\alpha$ is nonzero. If $n$ is even one has

$$
G_{0}^{\gamma}=|r|^{-\mu} N_{\mu}(\alpha|r|),
$$

where $N_{\mu}$ is the $\mu^{\text {th }}$ Neumann function. 
When $\alpha=0$, i.e. $\gamma=1$ we have the usual formula

$$
G_{0}(r)= \begin{cases}\log |r| & \text { when } \operatorname{rank} \mathbf{X}=2, \\ |r|^{(2-n)} & \text { otherwise } .\end{cases}
$$

When $\gamma<1$ it is possible to choose the fundamental solution $G_{0}^{\gamma}$ to be positive and decreasing at infinity:

$$
G_{0}^{\gamma}=\left(\frac{1}{z} \frac{\partial}{\partial z}\right)^{k} \exp (-\beta z)
$$

in odd-dimensional case with $n=2 k+1$ and

$$
G_{0}^{\gamma}=\left(\frac{1}{z} \frac{\partial}{\partial z}\right)^{k} K_{0}(\beta z)
$$

when $n=2 k+2$. Here $\beta=\sqrt{(1-\gamma)}|\rho|, z=|r|$ and $K_{0}$ is the modified Bessel function (see e.g. [56], p. 374).

Remark. The operator $\left(\frac{1}{z} \frac{\partial}{\partial z}\right)^{k}$ is the euclidean version of the shift operator for the hyperbolic space (9). The fact that it "shifts" the orders of the Bessel functions is known for a long time (see [55]).

Theorem 3.1. For given symmetric space $\mathbf{X}$ of noncompact type with even multiplicities the function

$$
G^{\gamma}(x, \xi)=C \mathscr{D}\left(r, \frac{\partial}{\partial r}\right) G_{0}^{\gamma}(r)
$$

where $r=r(x, \xi)$ are the radial coordinates of the point $x$ with respect to $\xi, \mathscr{D}$ is the shift operator (7), $G_{0}^{\gamma}$ is given above and $C$ is a certain constant, is a fundamental solution of Eq. (33). When $\gamma \leqq 1$ it is positive and decreasing at infinity.

The proof is based on the properties of the shift operator as well as in the previous section. The constant $C$ should be chosen in such a way that the whole expression has the same coefficient at the singularity as in the euclidean case (see e.g. [1]).

A fundamental solution decreasing at infinity is usually called the Green's function (for the precise definition and the proof of the existence of the Green's function for the general symmetric space of noncompact type, see [52]).

As a corollary we have the following two results, concerning the Laplace equation and its modified version.

Theorem 3.2. The Green's function of the Laplace equation $\mathscr{L}_{X} \phi=0$ on the odd-dimensional noncompact space $\mathbf{X}$ with even multiplicities has the following form:

$$
G(x, \xi)=C \mathscr{D}\left(r, \frac{\partial}{\partial r}\right)\left(\frac{1}{z} \frac{\partial}{\partial z}\right)^{k} \exp (-|\rho| z),
$$

where $z=|r(x, \xi)|, n=2 k+1$ is the rank of $\mathbf{X}$.

Remark. One can see that the corresponding function $G$ decreases at infinity exponentially. It can be considered as an analogue of the Newtonian potential on the corresponding symmetric space. It would be interesting to investigate the corresponding Kepler problem, we would expect some remarkable properties of the 
solutions for the symmetric spaces from List 1. For example, for the sphere and Lobachevsky space all orbits in this potential are known to be closed (see [57]).

For the odd-dimensional Lobachevsky space $H^{2 m+1}$ one has

$$
G(x, \xi)=C\left(\frac{1}{\sinh z} \frac{\partial}{\partial z}\right)^{m} \exp (-m z),
$$

$z=|r|$ is the distance between $x$ and $\xi$.

For $m=1$ one has the well-known formula

$$
G(x, \xi)=\frac{\exp (-|r|)}{4 \pi \sinh |r|}=\frac{1}{2 \pi \exp 2|r|-1}=\frac{1}{4 \pi} \text { coth }|r|-\text { const } .
$$

Example 2. For an odd-dimensional symmetric space of negative curvature dual to a compact simple Lie group we have the following Green's function (cf. [33,34]):

$$
G(x, \xi)=C \prod_{\alpha \in \mathscr{R}_{+}} \frac{(\alpha, r)}{\sinh (\alpha, r)}\left(\frac{1}{z} \frac{\partial}{\partial z}\right)^{k} \exp (-|\rho| z),
$$

$z=|r|$ is as before the distance between $x$ and $\xi$.

Remark. In this case $G$ depends not only on $|r|$ but on all radial coordinates $r$. The riemannian manifolds whose Laplacian has the fundamental solution $G(x, \xi)$ depending only on the distance between $x$ and $\xi$ are known as harmonic manifolds. A. Lichnerowicz [58] conjectured that all such manifolds are nothing else but the symmetric spaces of rank 1 . This is proved only under some assumptions $[58,59]$.

Let's consider now the modified Laplace equation

$$
\left(\mathscr{L}_{X}+|\rho|^{2}\right) \phi=0 \text {. }
$$

Theorem 3.3. The Green's function of the modified Laplace equation for a symmetric space $\mathbf{X}$ of noncompact type with even multiplicities has the form

$$
\hat{G}(x, \xi)=C \mathscr{D}\left(r, \frac{\partial}{\partial r}\right)|r|^{2-n}
$$

when $n=\operatorname{rank} \mathbf{X} \neq 2$ and

$$
\hat{G}(x, \xi)=C \mathscr{D}\left(r, \frac{\partial}{\partial r}\right) \log |r|
$$

for the spaces of rank 2.

Example. For the Lobachevsky space $\mathbf{X}=H^{2 m+1}$ we have

$$
\hat{G}(x, \xi)=C\left(\frac{1}{\sinh z} \frac{\partial}{\partial z}\right)^{m} z=C\left(\frac{1}{\sinh z} \frac{\partial}{\partial z}\right)^{m-1} \frac{1}{\sinh z},
$$

$z$ is the distance between $x$ and $\xi$. In particular for $\mathbf{X}=H^{3}$,

$$
\hat{G}(x, \xi)=\frac{1}{4 \pi \sinh |r(x, \xi)|} .
$$


Before consideration of the heat equation in the next section we would like to mention one more formula for the Green's function $G^{\gamma}$ in terms of the heat kernel $H(x, \xi, t)($ see $[52])$ :

$$
G^{\gamma}(x, \xi)=\int_{0}^{\infty} \exp \left(\gamma|\rho|^{2} t\right) H(x, \xi, t) d t .
$$

\section{Heat Kernels for the Symmetric Spaces with Huygens' Principle}

Consider the heat equation

$$
u_{t}=\mathscr{L}_{X} u \text {, }
$$

where $\mathscr{L}_{X}$ is the Laplace-Beltrami operator on the symmetric space $X=G / K$ with even root multiplicities $m_{\alpha}$. Here we find the explicit formulas for the fundamental solution of the Cauchy problem for Eq. (46). As well as for the wave equation the problem is to find the solution $F(x, t)$ of the equation

$$
H_{t}=\mathscr{L}_{X} H
$$

with the initial data

$$
\left.H\right|_{t=0}=\delta(x),
$$

where $\delta(x)$ is the delta function on $X$ concentrated in the "origin" $e K$ ( $e$ denotes the unit of the group $G$ ).

First we consider the noncompact case and let $X$ be a symmetric space of rank $n$ and $D$ be the corresponding shift operator given by Theorem 1.1. Define for $t>0$ the following function on $X$ :

$$
\begin{gathered}
H(x, t)=C\left(\frac{1}{2 \sqrt{\pi t}}\right)^{n} e^{-|\rho|^{2} t} D\left(r, \frac{\partial}{\partial r}\right) e^{-\frac{|r|^{2}}{4 t}}, \\
C=\left(-\frac{1}{2 \pi}\right)^{\frac{N-n}{2}}, \quad N=\operatorname{dim} X, \quad \rho=\frac{1}{2} \sum_{\alpha \in R_{+}} m_{\alpha} \alpha .
\end{gathered}
$$

Here $r \in \mathbf{R}^{n}$ is the radial component of the point $x=g K \in X$ determined from Cartan's decomposition (23). As well as it was for the wave equation the function (49) is well-defined on $X$.

Theorem 4.1. For any symmetric space $X$ of noncompact type with even root multiplicities the function (49) satisfies the heat equation (46) with the initial data $\left.H\right|_{t=0}=\delta(x)$ and defines the fundamental solution of Eq. (46) by the formula

$$
\Phi(x, \xi, t)=H\left(h^{-1} x, t\right) \quad \text { for } \xi=h K \in X, \quad h \in G .
$$

The proof of the theorem is similar to the proof of Theorem 2.1. First, it is easy to verify that the function $\varphi=\left(\frac{1}{2 \sqrt{\pi t}}\right)^{n} e^{-|\rho|^{2} t-\frac{|r|^{2}}{4 t}}$ satisfies the equation

$$
\varphi_{t}=\left(\Delta-|\rho|^{2}\right) \varphi
$$

where $\Delta=\sum_{i=1}^{n} \frac{\partial^{2}}{\partial r_{i}^{2}}$. Then the function $H(r)=C D\left(r, \frac{\partial}{\partial r}\right) \varphi$ satisfies Eq. (46) according to (7):

$$
\mathscr{L}_{X} H=\mathscr{L}_{X}^{\mathrm{rad}} H=C\left(\mathscr{L}_{X}^{\mathrm{rad}} \circ D\right) \varphi=C D \circ\left(\Delta-|\rho|^{2}\right) \varphi=(C D \varphi)_{t} .
$$


The initial conditions are also checked similar to the case of the wave equation. Indeed, if we present $H$ in accordance with (49) in the form

$$
\begin{aligned}
H(r)= & C\left(\frac{1}{\sqrt{\pi t}}\right)^{n} e^{-|\rho|^{2} t-\frac{|r|^{2}}{4 t}}\left(a_{0}(r)(4 t)^{-\frac{N-n}{2}}\right. \\
& \left.+a_{1}(r)(4 t)^{-\frac{N-n}{2}+1}+\cdots+a_{\frac{N-n}{2}}(r)\right),
\end{aligned}
$$

then the coefficients $a_{s}(r)$ will be the same as in (25). Thus $a_{s}(r)$ are regular and $a_{0}(r)$ tends to $(-2)^{\frac{N-n}{2}}$ while $r \rightarrow 0$. Now it is sufficient to note that

$$
\left.t^{s} e^{-\frac{|r|^{2}}{4 t}}\right|_{t=0}= \begin{cases}(2 \sqrt{\pi})^{N} \delta(x) & \text { for } 2 s=-N, \\ 0 & \text { for } 2 s>-N,\end{cases}
$$

and therefore $C\left(\frac{1}{2 \sqrt{\pi t}}\right)^{n} e^{-|\rho|^{2} t-\frac{|r|^{2}}{4 t}} a_{0}(r)(4 t)^{-\frac{N-n}{2}} \rightarrow \delta(x)$ for $t \rightarrow 0$. The theorem is proved.

Remark. The coincidence of the coefficients in the expansions of the fundamental solutions for the modified wave equation and for the heat equation is not accidental because of the following general result.

Let $M$ be an arbitrary complete riemannian manifold of an odd dimension $n$ and $L$ be a differential operator on $M$ which coincides in its main part with the Laplace-Beltrami operator.

Suppose now that the corresponding hyperbolic equation

$$
u_{t t}=L u
$$

satisfies Huygens' principle. In that case the fundamental solution of the Cauchy problem must have the following form in accordance with Hadamard's criterion:

$$
\Phi(x, \xi, t)=\sum_{j=0}^{p} V_{j}(x, \xi) \delta^{(p-\jmath)}(\Gamma)
$$

with $p=\frac{n-3}{2}$ and $\Gamma=t^{2}-r^{2}(x, \xi), r(x, \xi)$ is the distance between $x$ and $\xi$ on $M$. The coefficients $V_{j}$ are determined recursively and coincide up to some factors with the so-called Hadamard's coefficients (see e.g. [49]).

Consider now the corresponding "heat" equation

$$
u_{t}=L u,
$$

and the so-called parametrix for this equation, which is its formal solution of the form

$$
\mathscr{E}(x, \xi, t)=\frac{1}{(4 \pi t)^{\frac{n}{2}}} e^{-\frac{r^{2}(x, \xi)}{4 t}} \sum_{j=0}^{\infty} W_{j}(x, \xi)(4 t)^{j} .
$$

Theorem. If Eq. (52) satisfies Huygens' principle then the parametrix coefficients $W_{j}$ for the corresponding equation (54) coincide up to some constant factors with the coefficients $V_{j}$. In particular, $W_{j}=0$ for all $j>p$.

To prove this fact it is sufficient to compare the recursions for $V_{j}$ and for $W_{j}$ (see for instance [50]). 
Corollary. If Eq. (52) satisfies Huygens' principle, then the equation $u_{t t}=$ $L(u)+c u$ with arbitrary nonzero constant $c$ cannot satisfy this principle. In particular, the modified wave equation (2) on a symmetric space with even root multiplicities satisfies Huygens' principle only for the constant (3).

Indeed, the parametrices for such equations differ by the factor $e^{c t}$ and therefore their expansions cannot be finite for both equations.

Consider now the compact case. Let $X_{*}=U / K$ be a space of the compact type dual to the space of noncompact type $X=G / K$ and $\mathscr{R}$ be its root system. We may think of $U$ and $G$ as the subgroups of the complex group with the Lie algebra being the complexification of Lie algebras $\mathscr{U}$ and $\mathscr{G}$ of the groups $U, G$. Consider the decomposition $U=K A_{*} K$ of the group $U$ with $A_{*}=$ expi $\mathscr{A}$. This decomposition is dual to Cartan's decomposition (6) and it determines polar coordinates $(r, k)$ on $X_{*}$ in the similar way:

$$
u K=k \exp (\text { ir }) \mathrm{K}, \quad \text { where } \mathrm{k} \in \mathrm{K}, \mathrm{r} \in \mathscr{A} .
$$

Note that the radial coordinates $r=\left(r_{1}, \ldots, r_{n}\right)$ are defined only modulo the group $\hat{\mathbf{W}}$ generated by the Weyl group of $\mathscr{R}$ and the lattice $\hat{Q}=\{r \in \mathscr{A} \mid \exp ($ ir $) \in \mathrm{K}\}$. Assume now for simplicity that $U$ is simply connected and $K$ is connected. In that case the lattice $\hat{Q}$ is spanned by all elements of the form $\pi \alpha^{\vee}$ with $\alpha \in \mathscr{R}$, where $\alpha^{\vee}$ is dual to $\alpha: \alpha^{\vee}=2 \alpha /(\alpha, \alpha)$. In this situation the group $\hat{\mathbf{W}}$ is the affine Weyl group of the root system $\pi \mathscr{R}$ (see [43]).

Define now for $t>0$ the following function $\Theta(r, t)$ on $\mathscr{A} \cong \mathbf{R}^{n}$ :

$$
\Theta(r, t)=\Theta(r, t \mid \hat{Q})=\left(\frac{1}{2 \sqrt{\pi t}}\right)^{n} \sum_{\nu \in \hat{Q}} e^{-\frac{|r-v|^{2}}{4 t}},
$$

where $\hat{Q}$ is mentioned above: $\hat{Q}=\pi Q^{\vee}, Q^{\vee}$ is the dual root lattice for the system $\mathscr{R}$.

It is easy to see that $\Theta=\Theta(r, t \mid \hat{Q})$ satisfies the heat equation on the torus $T^{n}=\mathscr{A} / \hat{Q}$ :

$$
\Theta_{t}=\Delta \Theta
$$

where $\Delta=\frac{\partial^{2}}{\partial r_{1}^{2}}+\cdots+\frac{\partial^{2}}{\partial r_{n}^{2}}$ is the Laplacian on $T^{n}$. The initial data $\left.\Theta\right|_{t=0}=$ $\sum_{v \in \hat{Q}} \delta(r-v)$ is the Dirac $\delta$-function on the torus concentrated at the "zero." We can rewrite $\left.\Theta\right|_{t=0}$ as

$$
\left.\Theta\right|_{t=0}=\sum_{v \in \hat{Q}} \delta(r-v)=\sum_{p \in P} e^{2 i p(r)},
$$

where $P=\left\{p \in \mathscr{A}^{*} \mid p\left(\alpha^{\vee}\right) \in \mathbf{Z}\right.$ for all $\left.\alpha^{\vee} \in \mathscr{R}^{\vee}\right\}$ is the weight lattice for $\mathscr{R}$. Comparing the expansion (59) and Eq. (58) we see that the function (57) can be presented in the standard for the theta-functions form:

$$
\begin{aligned}
\Theta(r, t) & =\sum_{p \in P} e^{-4|p|^{2} t+2 i p(r)} \\
& =\sum_{N=\left(N_{1}, \ldots, N_{n}\right) \in \mathbf{Z}^{n}} \exp \left(\sum_{\mathrm{s}=1}^{\mathrm{n}} 2 \mathrm{i} \omega_{\mathrm{s}}(\mathrm{r}) \mathrm{N}_{\mathrm{s}}-4 \mathrm{t} \sum_{\mathrm{k}, 1}\left(\omega_{\mathrm{k}}, \omega_{1}\right) \mathrm{N}_{\mathrm{k}} \mathrm{N}_{1}\right) \\
& =\theta\left(2 \omega_{1}(r), \ldots, 2 \omega_{n}(r) \mid 4 i t \Omega\right),
\end{aligned}
$$


where $\omega_{1}, \ldots, \omega_{n}$ are the fundamental weights of $\mathscr{R}$ which form a basis of the weight lattice, and $\Omega$ is the Gram matrix for the fundamental weights: $\Omega_{k l}=\left(\omega_{k}, \omega_{l}\right)$ and the theta-function $\theta(z \mid B), z \in \mathbf{C}^{n}$ for a symmetric matrix $B$ with positive imaginary part is defined as

$$
\theta(z \mid B)=\sum_{N=\left(N_{1}, \ldots, N_{n}\right) \in \mathbf{Z}^{n}} \exp \left(\mathrm{i} \sum_{\mathrm{s}=1}^{\mathrm{n}} \mathrm{N}_{\mathrm{s}} \mathrm{z}_{\mathrm{s}}+\mathrm{i} \sum_{\mathrm{k}, 1} \mathrm{~B}_{\mathrm{kl}} \mathrm{N}_{\mathrm{k}} \mathrm{N}_{\mathrm{l}}\right) .
$$

Example. For $n=1$ and $T^{1}=\mathbf{R} / 2 \pi \mathbf{Z}$ we have $Q^{\vee}=2 \mathbf{Z}, P=\frac{1}{2} \mathbf{Z}, \omega_{1}=\frac{1}{2}$ and $\Theta(r, t)=\sum_{m \in \mathbf{Z}} e^{-m^{2} t+i m r}$ satisfies the heat equation on a circle with the initial data $\left.\theta\right|_{t=0}=\sum_{s \in \mathbf{Z}} \delta(r-2 \pi s)$ and coincides with one of the classical Jacobi's thetafunctions: $\Theta(r, t)=\theta(r \mid i t)$.

Now we are going to find the fundamental solution of the heat equation on the compact space $X_{*}=U / K$ with even root multiplicities. First we recall that as well as in the noncompact case the Laplace-Beltrami operator $\mathscr{L}_{X_{*}}$ has the radial part

$$
\mathscr{L}_{X_{*}}^{\mathrm{rad}}=\Delta+\sum_{\alpha \in \mathscr{R}_{+}} m_{\alpha} \cot (\alpha, r)\left(\alpha, \frac{\partial}{\partial r}\right),
$$

which can be reduced according to Theorem 1.1 to the form $\Delta+|\rho|^{2}$ by means of the corresponding shift operator $D\left(r, \frac{\partial}{\partial r}\right)$ :

$$
\mathscr{L}_{X_{*}}^{\mathrm{rad}} \circ D=D \circ\left(\Delta+|\rho|^{2}\right) .
$$

(We change the sign before $|\rho|^{2}$ because in the compact case the roots $\alpha \in \mathscr{A}^{*}$ should be restricted to the space $\mathscr{A}_{*}=i \mathscr{A}$.)

Define for $t>0$ the following function $H(r, t)$ on $X_{*}$ :

$$
H(r, t)=C e^{|\rho|^{2} t} D\left(r, \frac{\partial}{\partial r}\right) \Theta(r, t),
$$

where $\Theta(r, t)$ is theta-function (60) and $C$ is the same as in (49).

Theorem 4.2. Let $X_{*}=U / K$ be a symmetric space of the compact type with even root multiplicities, $U$ be simply-connected and $K$ be connected. Then the function (62) satisfies the heat equation (46) with the initial data $\left.H\right|_{t=0}=\delta(x)$ and determines the fundamental solution of the Cauchy problem for (46) by formula (50).

Proof. First note that the function (62) is well-defined on $X_{*}$. In fact, $\Theta(r, t)$ according to (57) is invariant under the affine Weyl group $\hat{\mathbf{W}}=W \times \pi Q^{\vee}$. The same is true for the operator $\mathscr{L}_{X_{*}}^{\text {rad }}$, for the highest symbol of $D$ :

$$
D_{0}\left(r, \frac{\partial}{\partial r}\right)=\prod_{\alpha \in \mathscr{R}_{+}}(\sin (\alpha, r))^{-\frac{1}{2} m_{\alpha}} \prod_{\alpha \in \mathscr{R}_{+}}\left(\alpha, \frac{\partial}{\partial r}\right)^{\frac{1}{2} m_{\alpha}},
$$

and hence for the whole $D\left(r, \frac{\partial}{\partial r}\right)$.

The fact that $H$ satisfies the heat equation follows from (58), (61) and (62) as well as in the noncompact case. The initial conditions also can be verified in a similar way.

Now consider the general formulas (49), (50), (62) for each case separately. 
1. For the odd-dimensional Lobachevsky space $X=H^{2 m+1}$ the formulas (9), (49) and (50) give:

$$
\Phi(x, \xi, t)=\frac{(-1)^{m} e^{-m^{2} t}}{2^{m+1} \pi^{m+\frac{1}{2}} \sqrt{t}}\left(\frac{1}{\sinh r} \frac{d}{d r}\right)^{m} e^{-\frac{r^{2}}{4 t}},
$$

where $r$ is the distance between $x$ and $\xi$.

2. For the symmetric spaces $X=G / K$ with a complex group of motions, all multiplicities $m_{\alpha}$ are equal to 2 and we have:

$$
\begin{aligned}
F(x, t) & =\frac{C e^{-|\rho|^{2} t}}{(2 \sqrt{\pi t})^{n}} \prod_{\alpha \in \mathscr{R}_{+}} \sinh ^{-1}(\alpha, r) \prod_{\alpha \in \mathscr{R}_{+}}\left(\alpha, \frac{\partial}{\partial r}\right) e^{-\frac{r^{2}}{4 t}} \\
& =\left(\frac{1}{2 \sqrt{\pi t}}\right)^{N} \prod_{\alpha \in \mathscr{R}_{+}} \frac{(\alpha, r)}{\sinh (\alpha, r)} e^{-\frac{r^{2}}{4 t}-|\rho|^{2} t}
\end{aligned}
$$

where $n$ and $N$ are respectively the rank and the dimension of $X, \rho=\sum_{\alpha \in \mathscr{R}_{+}} \alpha$. To get this formula we have used the following identity:

$$
\prod_{\alpha \in \mathscr{R}_{+}}\left(\alpha, \frac{\partial}{\partial r}\right) e^{\frac{r^{2}}{2}}=\prod_{\alpha \in \mathscr{R}_{+}}(\alpha, r) e^{\frac{r^{2}}{2}},
$$

which is similar to (27).

In particular, for $X=S L(n, C) / S U(n)$ we have similar to (28):

$$
F(h, t)=\left(\frac{1}{2 \sqrt{\pi t}}\right)^{n^{2}-1} \prod_{i<j} \frac{\left(r_{i}-r_{j}\right)}{\sinh \left(r_{i}-r_{j}\right)} \exp \left(-\frac{|r|^{2}}{4 t}-\frac{1}{3}\left(n^{3}-n\right) t\right) .
$$

3. For the noncompact space of AII type $X=S U^{*}(2 n) / S p(n)$ whose dimension is $N=(n-1)(2 n+1)$ we have similar to $(29)$ :

$$
\begin{aligned}
F(x, t)= & \left(\frac{1}{2 \sqrt{\pi}}\right)^{N} t^{\frac{1-n}{2}}\left(\prod_{i<j} \sinh ^{-2}\left(r_{i}-r_{j}\right)\right) e^{-\frac{4}{3}\left(n^{3}-n\right) t}(-2)^{\frac{n(n-1)}{2}} \\
& \times \mathscr{D}\left(\prod_{i<j}\left(r_{i}-r_{j}\right) e^{-\frac{|r|^{2}}{4 t}}\right)
\end{aligned}
$$

with $\mathscr{D}=\mathscr{D}_{n} \circ \mathscr{D}_{n-1} \circ \cdots \circ \mathscr{D}_{2}$ and $\mathscr{D}_{k}$ defined in (12)

4. For the noncompact space EIV of dimension 26 we obtain in accordance with the formulas of Sect. 1 for the shift operator the following expression for $F(r, t)$ :

$$
2^{-14} \pi^{-13} t^{-1}\left(\sinh r_{12} \sinh r_{23} \sinh r_{31}\right)^{-4} e^{-128 t} D_{3} \circ D_{2} \circ D_{1} \circ D_{0}\left(e^{-\frac{|r|^{2}}{4 t}}\right),
$$

where $r_{i j}=r_{i}-r_{j}, r_{1}+r_{2}+r_{3}=0$.

Now consider the compact case.

1. For the odd-dimensional sphere $X=S^{2 m+1}$ we have only one radial coordinate $r$ determined by the geodesic distance, $0 \leqq r \leqq \pi$. The function (60) for this case has the form

$$
\Theta(r, t)=\sum_{n \in \mathbf{Z}} e^{-n^{2} t+i n r}=\theta(r \mid i t),
$$


and the fundamental solution of the heat equation on $S^{2 m+1}$ is obtained by the formulas (9), (62):

$$
\Phi(x, \xi, t)=\frac{(-1)^{m}}{2^{1+m} \pi^{\frac{1}{2}+m}} e^{m^{2} t}\left(\frac{1}{\sin r} \frac{d}{d r}\right)^{m} \theta(r \mid i t),
$$

$r$ is the distance between $x$ and $\xi$ on a $S^{2 n+1}$.

2. Let $X$ be a compact simple simply-connected Lie group. In accordance with the formulas (10), (60), (62) we obtain:

$$
F(r, t)=C e^{|\rho|^{2} t}\left(\prod_{\alpha \in \mathscr{R}_{+}} \sin ^{-1}(\alpha, r)\right) \prod_{\alpha \in \mathscr{R}_{+}}\left(\alpha, \frac{\partial}{\partial r}\right) \Theta(r, t),
$$

where

$$
\Theta(r, t)=\sum_{p \in P} e^{-4|p|^{2} t+2 i p(r)},
$$

$P$ is the weight lattice for the root system $\mathscr{R}$ of $X$.

Thus $\Theta(r, t)$ is completely determined by the weight lattice $P(\mathscr{R}) \subset \mathscr{A}^{*} \cong \mathbf{R}^{n}$ which can have one of the following form:

1) The standard cubic lattice $P\left(C_{n}\right)=\mathbf{Z}^{n}$.

2) The "chess" lattice $P\left(B_{n}\right)=P\left(D_{n}\right)=\mathbf{Z}^{n} \oplus \mathbf{Z}\left(\frac{1}{2} \sum_{i=1}^{n} e_{i}\right)$.

3) The orthogonal projection $P\left(A_{n}\right)$ of the cubic lattice $\mathbf{Z}^{n+1} \subset \mathbf{R}^{n+1}$ to the hyperplane $x_{1}+\cdots+x_{n+1}=0, P\left(A_{n}\right)$ is isomorphic to the lattice of integer vectors with zero sum of their components.

4) $P\left(F_{4}\right)=P\left(D_{4}\right) \subset \mathbf{R}^{4}$.

5) $P\left(G_{2}\right) \cong P\left(A_{2}\right) \subset \mathbf{R}^{2}$.

6) The special lattices $P\left(E_{i}\right), i=6,7,8$. For instance, $P\left(E_{8}\right) \subset \mathbf{R}^{8}$ consists of all $\xi=\left(\xi_{1}, \ldots, \xi_{8}\right)$ with even $\sum_{i=1}^{8} \xi_{i}$ and all $\xi_{i}$ being simultaneously integers or half-integers.

For all these lattices the theta-function (63) can be expressed in terms of classical one-dimensional theta-functions. Now we show it for the classical lattices only.

1) $P=\mathbf{Z}^{n}$. It is clear that in this case $\Theta(r, t)=\prod_{j=1}^{n} \theta\left(2 r_{j} \mid 4 i t\right)$, where $\theta(x \mid \tau)=$ $\sum_{n \in \mathbf{Z}} e^{i \tau n^{2}+i n x}$ denotes the classical one-dimensional theta-function.

2) $P=\mathbf{Z}^{n} \oplus \mathbf{Z}\left(\frac{1}{2} \sum_{i=1}^{n} e_{i}\right)$. In that case we have:

$$
\Theta(r, t)=\sum_{p \in \mathbf{Z}^{n}} e^{-4|p|^{2} t+2 i p(r)}+\sum_{p \in \mathbf{Z}^{n}} e^{-4\left|p+p_{0}\right|^{2} t+2 i\left(p+p_{0}\right)(r)},
$$

where $p_{0}=\frac{1}{2} \sum_{i=1}^{n} e_{i}$.

Thus

$$
\Theta(r, t)=\prod_{j=1}^{n} \theta\left(2 r_{j} \mid 4 i t\right)+e^{-4 t\left|p_{0}\right|^{2}+2 i p_{0}(r)} \prod_{j=1}^{n} \theta\left(2 r_{j}+4 i t \mid 4 i t\right)
$$


3) $P=\mathbf{Z}^{n+1} \cap\left\{x_{1}+\cdots+x_{n+1}=0\right\}$. Let $\theta(x \mid \tau)$ denote as before $\theta(x \mid \tau)=$ $\sum_{k \in \mathbf{Z}} e^{i k^{2} \tau+i k x}$. Then

$$
\begin{aligned}
f(x \mid \tau) & =\sum_{k=0}^{n} \prod_{j=1}^{n+1} \theta\left(x_{j}+\frac{2 k \pi}{n+1} \mid \tau\right)=\sum_{k=0}^{n} \prod_{j=1}^{n+1} \sum_{m_{j} \in \mathbf{Z}} e^{i m_{j}^{2} \tau+i m_{j}\left(x_{j}+\frac{2 k \pi}{n+1}\right)} \\
& =\sum_{k=0}^{n} \sum_{\left(m_{1}, \ldots, m_{n+1}\right) \in \mathbf{Z}^{n+1}} e^{i|m|^{2} \tau+i(m, x)+\frac{2 k i \pi}{n+1}\left(m_{1}+\cdots+m_{n+1}\right)},
\end{aligned}
$$

where $(m, x)=\sum m_{i} x_{i},|m|^{2}=m_{1}^{2}+\cdots+m_{n+1}^{2}$.

Then changing the order in summation we get:

$$
f(x)=\sum_{l \in \mathbf{Z}} \sum_{m_{1}+\cdots+m_{n+1}=l(n+1)}(n+1) e^{i|m|^{2} \tau+i(m, x)} .
$$

Put $k_{i}=m_{i}-l$, then $m=k+l \varepsilon$ and for $k_{1}+\cdots+k_{n+1}=0 \quad|m|^{2}=|k|^{2}+l^{2}|\varepsilon|^{2}$ with $\varepsilon=(1, \ldots, 1)$. So

$$
\begin{aligned}
f(x) & =\sum_{l \in \mathbf{Z}} \sum_{k_{1}+\cdots+k_{n+1}=0}(n+1) e^{i l^{2}(n+1) \tau} \cdot e^{i|k|^{2} \tau+i(k, x)+i l(\varepsilon, x)} \\
& =(n+1) \sum_{l \in \mathbf{Z}} e^{i l^{2}(n+1) \tau+i l\left(x_{1}+\cdots+x_{n+1}\right)} \sum_{k_{1}+\cdots+k_{n+1}=0} e^{i|k|^{2} \tau+i(k, x)} \\
& =(n+1) \theta\left(x_{1}+\cdots+x_{n+1} \mid(n+1) \tau\right) \cdot \sum_{k_{1}+\cdots+k_{n+1}=0} e^{i|k|^{2} \tau+i(k, x)} .
\end{aligned}
$$

The last sum is the required many-dimensional theta-function that corresponds to the lattice $P \in \mathbf{Z}^{n+1} \cap\left\{x_{1}+\cdots+x_{n+1}=0\right\}$. Thus for $\mathscr{R}=A_{n}$ the function (60) has the form

$$
\begin{aligned}
\Theta(r, t)= & \sum_{k_{1}+\cdots+k_{n+1}=0} e^{-4 t|k|^{2}+2 i(k, r)} \\
= & {\left[(n+1) \theta\left(2 r_{1}+\cdots+2 r_{n+1} \mid 4 i(n+1) t\right)\right]^{-1} } \\
& \times \sum_{k=0}^{n} \prod_{j=1}^{n+1} \theta\left(2\left(r_{j}+\frac{k \pi}{n+1}\right) \mid 4 i t\right)
\end{aligned}
$$

Finally, because $r_{1}+\cdots+r_{n+1}=0$,

$$
\Theta(r, t)=\frac{1}{(n+1) \theta(0 \mid 4 i(n+1) t)} \sum_{k=0}^{n} \prod_{j=1}^{n+1} \theta\left(2\left(r_{j}+\frac{k \pi}{n+1}\right) \mid 4 i t\right) .
$$

It is useful to rewrite this theta-function for the isomorphic lattice which is the orthogonal projection of the standard cubic lattice in $\mathbf{R}^{n}$ to the hyperplane $r_{1}+\cdots+r_{n}=0$. The result is the following:

$$
\begin{aligned}
\Theta(r, t)= & \frac{1}{n \theta(0 \mid 2 i(n-1) t)} \\
& \times \sum_{k=0}^{n-1} \prod_{j=1}^{n} \theta\left(\frac{(n-1)\left(r_{j}-r_{j+1}\right)}{n}+\frac{2 k \pi}{n} \mid \frac{2 i(n-1) t}{n}\right)
\end{aligned}
$$

(in this formula $r_{j+1}$ for $j=n$ is $r_{1}$ ). 
As a result, the fundamental solution for the heat equation on a compact simple simply-connected Lie group can be obtained by substitution of the corresponding expression for the function (60) to (62).

Example. For $\mathbf{X}=\mathbf{S U}(n)$ the radial coordinates $r_{1}, \ldots, r_{n}$ of a unitary matrix $x \in$ $\mathbf{S U}(n)$ are the logarithms of its eigenvalues divided by $i, r_{1}+\cdots+r_{n}=0$. The roots $\alpha \in \mathscr{R}$ have the form $\frac{r_{i}-r_{j}}{2}$ and the heat kernel can be obtained from the formulas (62), (65):

$$
\begin{aligned}
F(x, t)= & C \exp \left(\frac{1}{12}\left(n^{3}-n\right) t\right) \frac{1}{n \theta(0 \mid-(n-1) t / 2)}\left(\prod_{i<j} 2 \sin \frac{r_{i}-r_{j}}{2}\right)^{-1} \\
& \times \prod_{i<j}\left(\frac{\partial}{\partial r_{i}}-\frac{\partial}{\partial r_{j}}\right) \sum_{k=0}^{n-1} \prod_{j=1}^{n} \theta\left(\frac{(n-1)\left(r_{j}-r_{j+1}\right)}{2 n}+\frac{2 k \pi}{n} \mid i \frac{(n-1) t}{2 n}\right) .
\end{aligned}
$$

At last for the simply-connected compact spaces AII and EIV the corresponding fundamental solutions are obtained by the substitution of (65) into the formula (62).

\section{Shift Operators for $\mathrm{BC}_{n}$ and Symmetric Spaces}

In this section we consider the class of the symmetric spaces characterized by the following properties: 1) their root system $\mathscr{R}$ is of $\mathbf{B C}_{n}$ type, 2) if we identify $\mathscr{R}$ with the set $\left\{ \pm e_{i}, \pm 2 e_{i}, \pm e_{i} \pm e_{j}\right\}$ in $\mathbf{R}^{n}$, then the roots $\pm e_{i} \pm e_{j}$ have even multiplicity. We have listed all such spaces in the Introduction (see List 2).

For these spaces the radial part of their Laplace-Beltrami operator can be reduced to a rather simple form with the help of a suitable shift operator. This allows us to give some formulas for the fundamental solutions of the modified wave, Laplace and heat equations on such a space.

We will need the following facts about the shift operators for the root systems of the type $\mathscr{R}=B C_{n}$. We use the notations of Sect. 1 and consider the noncompact case mainly.

Consider now the root system $\mathscr{R}=B C_{n}$ consisting of the vectors $\pm e_{i}, \pm 2 e_{i}$, $\pm e_{i} \pm e_{j}$ in $\mathbf{R}^{n}$. We have three orbits for the Weyl group $\mathbf{W}$ in $\mathscr{R}: S_{1}=\left\{ \pm e_{i}\right\}$, $S_{2}=\left\{ \pm 2 e_{i}\right\}, S_{3}=\left\{ \pm e_{i} \pm e_{j}\right\}_{i<j}$. Thus any $\mathbf{W}$-invariant function $k=\left(k_{\alpha}\right)$ on $\mathscr{R}$ is determined by three numbers and the corresponding operator (15) $\mathscr{L}_{k}$ for given $k=\left(k_{1}, k_{2}, k_{3}\right)$ has the form

$$
\begin{aligned}
\mathscr{L}_{k}= & \Delta+\sum_{i=1}^{n}\left(2 k_{1} \operatorname{coth} r_{i}+4 k_{2} \operatorname{coth} 2 r_{i}\right) \frac{\partial}{\partial r_{i}}+2 k_{3} \sum_{i<j}\left(\operatorname{coth}\left(r_{i}-r_{j}\right)\left(\frac{\partial}{\partial r_{i}}-\frac{\partial}{\partial r_{j}}\right)\right. \\
& \left.+\operatorname{coth}\left(r_{i}+r_{j}\right)\left(\frac{\partial}{\partial r_{i}}+\frac{\partial}{\partial r_{j}}\right)\right)+|\rho(k)|^{2} .
\end{aligned}
$$

As well as in Sect. 1 we denote by $D_{k, S}$ the operator (14) for a given W-orbit $S$ in $\mathscr{R}$ and $k=\left(k_{1}, k_{2}, k_{3}\right)$. Define now for $l=(0,0,1),(0,1,0)$ and $(2,-1,0)$ the following differential operators $\mathscr{D}_{l}$ in $\mathbf{R}^{n}$ depending on $k=\left(k_{1}, k_{2}, k_{3}\right)$ :

$$
\begin{gathered}
\mathscr{D}_{(0,0,1)}=D_{k, S_{3}}, \quad \mathscr{D}_{(0,1,0)}=D_{k, S_{2}}, \\
\mathscr{D}_{(2,-1,0)}=\pi^{3-2 k_{2}} \circ D_{\tilde{k}, S_{2}} \circ \pi^{2 k_{2}-1},
\end{gathered}
$$

where $k=\left(k_{1}, k_{2}, k_{3}\right), \tilde{k}=\left(k_{1}+2 k_{2}-1,1-k_{2}, k_{3}\right), \pi=\pi(r)=\prod_{i=1}^{n} \frac{\sinh 2 r_{i}}{\sinh r_{l}}$. 
Theorem 5.1. [45]. Differential operator (67) $\mathscr{D}_{l}=\mathscr{D}_{l}(k)$ shifts the operator (66) from $k$ to $k+l$ in the following sense:

$$
D_{l}(k) \circ \mathscr{L}_{k}=\mathscr{L}_{k+l} \circ D_{l}(k) .
$$

Remark 1. For $\mathscr{R}=B C_{1}$ we have only two $\mathbf{W}$-orbits, two corresponding shift operators

$$
\begin{aligned}
D_{(0,1)}(k) & =\frac{2}{\sinh 2 r} \frac{d}{d r}, \\
D_{(2,-1)}(k) & =\frac{\sinh 2 r}{2 \sinh ^{2} r} \frac{d}{d r}+2 k_{2}-1,
\end{aligned}
$$

and the relations (68) have the form

$$
\begin{gathered}
D_{(0,1)} \circ \mathscr{L}_{k_{1}, k_{2}}=\mathscr{L}_{k_{1}, k_{2}+1} \circ D_{(0,1)}, \\
D_{(2,-1)} \circ \mathscr{L}_{k_{1}, k_{2}}=\mathscr{L}_{k_{1}+2, k_{2}-1} \circ D_{(2,-1)},
\end{gathered}
$$

with

$$
\mathscr{L}_{k_{1}, k_{2}}=\frac{d^{2}}{d r^{2}}+\left(k_{1} \operatorname{coth} r+2 k_{2} \operatorname{coth} 2 r\right) \frac{d}{d r}+\left(k_{1}+2 k_{2}\right)^{2} .
$$

Remark 2. For the special case $k=\left(k_{1}, k_{2}, 0\right)$ the shift operators (67) can be described in a more effective way using the following facts.

1) If $k=\left(k_{1}, k_{2}, 0\right)$ then $\mathscr{L}_{k}$ is in fact a sum of commuting one-dimensional operators of the form (71) and therefore $\mathscr{D}_{(0,1,0)}$ and $\mathscr{D}_{(2,-1,0)}$ can be presented as a composition of the operators (70):

$$
\begin{aligned}
\mathscr{D}_{(0,1,0)} & =\prod_{i=1}^{n} D_{(0,1)}\left(r_{i}, \frac{\partial}{\partial r_{i}}\right), \\
\mathscr{D}_{(2,-1,0)} & =\prod_{i=1}^{n} D_{(2,-1)}\left(r_{i}, \frac{\partial}{\partial r_{i}}\right) .
\end{aligned}
$$

2) If $\mathscr{J}$ denotes the operator of multiplication by the function

$$
J(r)=\prod_{i<j}\left(\sinh ^{2} r_{i}-\sinh ^{2} r_{j}\right),
$$

then we have the following relation between $\mathscr{L}_{k_{1}, k_{2}, 0}$ and $\mathscr{L}_{k_{1}, k_{2}, 1}$ in accordance with the observation by Berezin and Karpelevich [53]:

$$
\mathscr{J} \circ \mathscr{L}_{k_{1}, k_{2}, 1}=\mathscr{L}_{k_{1}, k_{2}, 0} \circ \mathscr{J} .
$$

It is easy to deduce from this fact that the shift operator $(67) \mathscr{D}_{(0,0,1)}$ can be presented for $k=\left(k_{1}, k_{2}, 0\right)$ in the form

$$
\mathscr{D}_{(0,0,1)}=J^{-1} \prod_{i<j}\left(\mathscr{L}_{k_{1}, k_{2}}^{2}\left(r_{i}, \frac{\partial}{\partial r_{i}}\right)-\mathscr{L}_{k_{1}, k_{2}}^{2}\left(r_{j}, \frac{\partial}{\partial r_{j}}\right)\right),
$$

where $\mathscr{L}_{k_{1}, k_{2}}$ is the operator (71).

Definition. Let $\mathbf{X}$ be a symmetric space of rank $n$ from List 2. If $\mathbf{X}$ is not of the type AII with odd $p+q$ define the space $\mathbf{X}_{0}$ as the following product of 
spheres of the radius 1 if $k_{1}$ is halfinteger and of radius $1 / 2$ if $k_{2}$ is halfinteger, or corresponding hyperbolic planes:

$$
\begin{aligned}
& \mathbf{X}=\mathbf{S}^{2} \times \cdots \times \mathbf{S}^{2} \text { (n times) in the compact case, } \\
& \mathbf{X}=\mathbf{H}^{2} \times \cdots \times \mathbf{H}^{2} \text { (n times) in the noncompact case. }
\end{aligned}
$$

For the case AIII with odd $p+q$ one should replace $\mathbf{S}^{2}$ and $\mathbf{H}^{2}$ by the complex projective plane $\mathbf{C P}^{2}$ and its dual hyperbolic version $\mathbf{C H}^{2}$ correspondingly.

Recall (see Sect. 3) that for an arbitrary symmetric space $\mathbf{X}$ the modified Laplace-Beltrami operator $\hat{\mathscr{L}}_{X}$ is defined as $\mathscr{L}_{X}-c$, where $\mathscr{L}_{X}$ is the usual Laplace-Beltrami operator and $c$ is of the form (3). In particular, for the spaces from List 2 the radial part of the modified Laplace-Beltrami operator has the form (66).

For the spaces from List 2 the following analogue of Theorem 1.1 is true.

Theorem 5.2. Let $\mathbf{X}$ be a symmetric space of rank $n$ from List 2 and $\mathbf{X}_{0}$ be the corresponding space defined above. Then the radial parts of the modified LaplaceBeltrami operators on $\mathbf{X}$ and $\mathbf{X}_{0}$ can be reduced one to another by means of a suitable shift operator $\mathscr{D}$ :

$$
\hat{\mathscr{L}}_{X}^{\mathrm{rad}} \circ \mathscr{D}=\mathscr{D} \circ \hat{\mathscr{L}}_{X_{0}}^{\mathrm{rad}} .
$$

For the proof of the theorem it is sufficient to check in each case except AIII with odd $p+q$ that the suitable composition of "elementary" shifts operators (67) reduces $k_{1}, k_{2}, k_{3}$ in the operator (66) to $1 / 2,0,0$ or to $0,1 / 2,0$ for which this operator is in fact $\mathscr{L}_{X_{0}}^{\text {rad }}+c_{0}$. In the case AIII with odd $p+q$ the operator $\mathscr{L}_{q-p, 1 / 2,1}$ similarly can be reduced to $\mathscr{L}_{1,1 / 2,0}$.

Remark. It is easy to see that the shift operator $\mathscr{D}(r, \partial / \partial r)$ constructed in Theorem 4.2 has the highest symbol

$$
\prod_{\alpha \in \mathscr{R}_{+}}(\sinh (\alpha, r))^{-\left[k_{\alpha}\right]} \prod_{\alpha \in \mathscr{R}_{+}}\left(\alpha, \frac{\partial}{\partial r}\right)^{\left[k_{\alpha}\right]},
$$

where $\left[k_{\alpha}\right]$ is the integer part of $k_{\alpha}$ except the case AIII with odd $p+q$ in which for $\alpha=e_{i}\left[k_{\alpha}\right]$ should be replaced by $k_{\alpha}-1$.

Now the fundamental solutions of the Cauchy problem for the modified wave equation and heat equation as well as the Green's functions on symmetric spaces of type AIII, CII, DIII, EIII, EVII and FII can be described in the following way.

Theorem 5.3. Let $\mathbf{X}$ be a space from List 2 and $\mathbf{X}_{0}$ be the corresponding space defined above. The fundamental solutions of the corresponding modified wave equations $F$ and $F_{0}$ are related by the shift operator $\mathscr{D}(r, \partial / \partial r)$ constructed in Theorem 5.2:

$$
F(r, t)=C_{1} \mathscr{D} F_{0}(r, t) .
$$

For the heat kernel and the Green's function on $\mathbf{X}$ in noncompact case one has the following relations

$$
\begin{aligned}
G(r) & =C_{2} \mathscr{D} G_{0}^{\gamma}(r), \\
H(r, t) & =C_{3} e^{\left(c-c_{0}\right) t} \mathscr{D} H_{0}(r, t),
\end{aligned}
$$

where $c_{0}$ and $c$ are the constants (3) for $\mathbf{X}_{0}$ and $\mathbf{X}, \gamma=1-\frac{c}{c_{0}}, G_{0}^{\gamma}$ is the fundamental solution of the corresponding equation (3) on $\mathbf{X}_{0}, C_{1}, C_{2}$ and $C_{3}$ are some constants. 
The proof of the theorem is similar to the case of the spaces with even root multiplicities.

The heat kernel for a direct product of some riemannian manifolds is essentially the product of the corresponding heat kernels. For the wave and Laplace equation there is also some procedure giving the fundamental solution on the product if it is known for each factor (see e.g. [54]), which is the case in our situation.

For example, the fundamental solution for the modified wave equation on the two-dimensional sphere has the form:

$$
\Phi_{0}(x, \xi, t)=C \frac{\theta\left(t^{2}-r^{2}\right)}{\sqrt{\cos r-\cos t}},
$$

where $r$ is the distance between $x$ and $\xi, \theta$ is Heaviside function. This implies the following formula for the even-dimensional sphere $\mathbf{S}^{2 n}$ :

$$
\Phi(x, \xi, t)=C\left(\frac{1}{\sin r} \frac{d}{d r}\right)^{n-1} \frac{\theta\left(t^{2}-r^{2}\right)}{\sqrt{\cos r-\cos t}},
$$

with some constant $C$.

For the modified wave equation on the complex projective plane $\mathbf{C} P^{2}$ one has (see e.g. [60])

$$
\Phi(x, \xi, t)=C \frac{1}{\sin t} \frac{d}{d t}\left(\frac{\theta\left(t^{2}-r^{2}\right)}{\sqrt{\cos 2 r-\cos 2 t}}\right),
$$

where $r$ is the distance between $x$ and $\xi$ and the similar formula is true for $\mathbf{C} H^{2}$.

Green's functions for all rank 1 symmetric spaces can be found in the last chapter of Helgason's book [18].

Acknowledgements. This work was partially supported by Soros International Science Foundation (grant MD-8000), INTAS grant (INTAS-93-0166) and Russian Foundation for Fundamental Research (grant 94-01-01444).

We are grateful to Prof. S. Helgason for sending us some of his papers, to G. Olshansky for pointing out the paper [53] and to V. Kargin, who presented to us some calculations with thetafunctions. One of us (A.V.) would like to thank V. Fateev, P. Lax, J. Moser and Y. Pinchover for useful discussions and Forschungsinstitut für Mathematik (ETH, Zürich) for the hospitality during the summer term of 1995.

Note added in proof. Recently we learned from T. Branson about the papers [62], [63], where another proof of the Huygens' Principle for the modified wave equations on the symmetric spaces with even multiplicities is found both in compact and noncompact cases. We are grateful to Prof. Branson for sending us the copies of these interesting papers.

\section{References}

1. Courant, R., Hilbert, D.: Methods of Mathematical Physics. Vol. 2. New York: 1964

2. Hadamard, J.: Lectures on Cauchy's problem. New Haven, CT: Yale Univ. Press, 1923

3. Günther, P.: Huygens' Principle and Hyperbolic Equations. Boston: Acad. Press. 1988

4. Berest, Yu.Yu., Veselov, A.P.: Huygens' Principle and Coxeter Groups. Uspekhi Mat. Nauk. 48(3), 181-182 (1993)

5. Berest, Yu.Yu., Veselov, A.P.: Hadamard's Problem and Coxeter Groups: New examples of Huygens' equations. Funk. Analiz i ego pril. 28(1), 3-15 (1994) 
6. Lagnese, J.E., Stellmacher, K.L.: A method of generating classes of Huygens' operators. J. Math. Mech. 17, 5, 461-472 (1967)

7. Lagnese, J.E.: A solution of Hadamard's Problem for a restricted class of operators. Proc. Am. Math. Soc. 19, 981-988 (1968)

8. Dubrovin, B.A., Matveev, V.B., Novikov, S.P.: Nonlinear KdV-type equations, finite-gap linear operators and Abelian varieties. Uspekhi Mat. Nauk. 31(1), 59-164 (1976)

9. Dubrovin, B.A., Krichever, I.M., Novikov, S.P.: Schrödinger equation in periodic field and riemannian surfaces. Sov. Mat. Dokl. 229, 15-18 (1976)

10. Krichever, I.M.: The methods of algebraic geometry in the theory of nonlinear equations. Uspekhi Mat. Nauk. 32(6), 198-245 (1977)

11. Veselov, A.P., Novikov, S.P.: Finite-gap two-dimensional periodic Schrödinger operators: potential operators. Sov. Mat. Dokl. 279, 784-788 (1984)

12. Chalykh, O.A., Veselov, A.P.: Commutative rings of partial differential operators and Lie algebras. Commun. Math. Phys. 126, 597-611 (1990)

13. Feldman, J., Knörrer, H., Trubowitz, E.: There is no two-dimensional analogue of Lamé's equation. Math. Ann. 294, 295-324 (1992)

14. Calogero, F.: Solution of the one-dimensional $n$-body problem with quadratic and/or inversely quadratic pair potential. J. Math. Phys. 12, 419-436 (1971)

15. Olshanetsky, M.A., Perelomov, A.M.: Quantum integrable systems related to Lie algebras. Phys. Rep. 94, 313-404 (1983)

16. Chalykh, O.A., Veselov, A.P.: Integrability in the theory of the Schrödinger operator and harmonic analysis. Commun. Math. Phys. 152, 29-40 (1993)

17. Veselov, A.P., Styrkas, K.L., Chalykh, O.A.: Algebraic integrability for Schrödinger equation and finite reflection groups. Teoret. i mat. fizika. 94(2), 253-275 (1993)

18. Helgason, S.: Differential geometry, Lie groups and symmetric spaces. New York: Academic Press, 1978

19. Postnikov, M.M.: Lie groups and Lie algebras. Moscow: "Nauka", 1982

20. Veselov, A.P., Chalykh, O.A.: Explicit formulas for the spherical functions of symmetric spaces of AII type. Funk. Analiz i ego pril. 26(1), 74-76 (1992)

21. Helgason, S.: Some results on Radon transforms, Huygens' principle and X-ray transforms. Contemp. Math. 63, 151-177 (1987)

22. Olevski, M.N.: Solution du problème de Cauchy pour l'equation des un espace à $\mathrm{n}$ dimensions a courbure constante. Sov. Mat. Dokl. 46, C.3-6 (1945)

23. Günther, P.: Uber einige specialle Probleme aus der Theorie der linearen partialen Differentialgleichungen zweiter Ordnung. Ber. Verh. Sachs. Akad. Wiss, 102, 1957, pp. 1-50

24. Lax, P.D., Phillips, R.S.: An example of Huygens' Principle. Comm. Pure and Appl. Math. 31, 415-421 (1978)

25. Solomatina, L.E.: Translation presentation and Huygens' principle for invariant wave equation on riemannian symmetric space. Izvestia vuzov. 30, 70-74 (1986)

26. Olafsson, G., Schlichtkrull, H.: Wave propagation on Riemannian symmetric spaces. J. Funct. Anal. 107, 270-278 (1992)

27. Helgason, S.: Huygens' Principle for wave equations on Symmetric spaces. J. Funct. Anal. 107, 279-288 (1992)

28. Helgason, S.: Wave equations on homogeneous spaces. Lect. Notes in Math. 1077, Berlin: Springer-Verlag, 1984, pp. 252-287

29. Helgason, S.: Solvability questions for invariant differential operators. In: "Group Theoretical Methods in Physics". (Montreal) New York: Academic Press, 1977

30. Semenov-Tian-Shanski, M.A.: Harmonic analysis on riemannian symmetric spaces of negative curvature and scattering theory. Sov. Mat. Izvestia. 40(3), 562-592 (1976)

31. Dowker, J.S.: Quantum Mechanics on Group Space and Huygens' Principle. Ann. Phys. 62, 361-382 (1971)

32. Chalykh, O.A., Veselov, A.P.: Hadamard problem for the wave equations on symmetric spaces. To appear

33. Dynkin, E.B.: Nonnegative eigenfunctions of the Laplace-Beltrami operator and the Brownian motion in some symmetric spaces. Dokl. Akad. Nauk. 141, 288-291 (1961)

34. Nolde, E.L.: Nonnegative eigenfunctions of the Laplace-Beltrami operator on symmetric spaces with a complex group of motion. Uspekhi Mat. Nauk. 21, n.5, 260-262 (1966)

35. Kiprijanov, I.A., Ivanov, L.A.: Euler-Poisson-Darboux equation on riemannian space. Sov. Mat. Dokl. 260(4), 790-794 (1981) 
36. Kiprijanov, I.A., Ivanov, L.A.: Cauchy problem for Euler-Poisson-Darboux equation on homogeneous symmetric riemannian space. Trudy Math. Inst. Steklov 170, (1984)

37. Eskin, L.D.: Heat equation and Weierstrass transformation on some symmetric spaces. Izvestia vuzov. 5, 151-165 (1965)

38. Fegan, H.D.: The fundamental solution of the heat equation on a compact Lie group. J. Differ. Geom. 18, 659-668 (1983)

39. Lu, Q.-K.: The heat kernels of symmetric spaces. Proc. of Symposia in pure Math. 54, Part 2, (1993) pp. 401-409

40. Gelfand, I.M., Shilov, G.E.: Generalized functions. Vol. 1, New York: Academic Press, 1964

41. Berezin, F.A.: Laplace-Beltrami operators on semisimple Lie groups. Ann. Moscow Math. Soc. 6, 371-463 (1957)

42. Chalykh, O.A.: Commuting maps and differential operators connected with Lie algebras (thesis). Moscow State University, 1992

43. Helgason, S.: Groups and geometric analysis. New York: Academic Press, 1984

44. Opdam, E.: Root systems and hypergeometric functions IV. Compositio Math. 67, 191-209 (1988)

45. Heckman, G.: An elementary approach to hypergeometric shift operators of Opdam. Invent. Math. 103, 341-350 (1991)

46. Dunkl, C.: Differential-difference operators associated to reflection groups. Trans. Am. Math. Soc. 311, 181-191 (1989)

47. Vretare, L.: Formulas for elementary spherical functions and generalized Jacobi polynomials. SIAM J. Math. An. 15, No 4, 805-833 (1984)

48. Beerends, R.: On the Abel transformations and its inversion. Comp. Math. 66, 145-197 (1988)

49. Berest, Yu.Yu., Veselov, A.P.: Huygens' principle and Integrability. Uspekhi mat. nauk. 49(6), 7-78 (1994)

50. Minakshisundaram, S., Pleijel, A.: Some properties of the eigenfunctions of the Laplace operator on Riemann manifolds. Canadian J. Math. 1, 242-256 (1949)

51. Weinstein, A.: On the wave equation and the equation of Euler-Poisson. Proc. Simpos. Appl. Math. 5, 137-147 (1954)

52. Karpelevich, F.I.: The geometry of geodesics and eigenfunctions of the Laplace-Beltrami operator on symmetric spaces. Trans. Moscow Math. Soc. 14, 48-185 (1965)

53. Berezin, F.A., Karpelevic̀, F.I.: Zonal spherical functions and Laplace operators for some symmetric spaces. Sov. Mat. Dokl. 118(1), 9-12 (1958)

54. Lagnese, J.: The fundamental solution and Huygens' principle for decomposable differential operators. Arch. Rat. Mech. and Anal. 19(4), 299-307 (1965)

55. Whittaker, E.T., Watson, G.N.: A course of modern Analysis. Cambridge: Cambridge Univ. Press, 1920

56. Abramowitz, M., Stegun, I.: Handbook of Mathematical functions. New York: Dover, 1968

57. Kozlov, V.V., Harin, A.O.: Kepler's problem in constant curvature spaces. Celest. Mech. and Dynam. Astron. 54, 393-399 (1992)

58. Lichnerowicz, A.: Sur les espaces riemanniens complement harmoniques. Bull. Soc. Math. France. 72, 146-168 (1994)

59. Szabo, Z.I.: The Lichnerowicz conjecture on harmonic manifolds. J. Diff. Geom. 31, 1-28 (1990)

60. Bunke, U., Olbrich, M.: The wave kernel for the Laplacian on locally symmetric spaces of rank one, theta functions, trace formulas and the Selberg zeta function. Preprint, March 1994, Humboldt Univ. Berlin

61. Helgason, S.: Geometric Analysis on Symmetric Spaces. AMS Math. Surveys and Monographs. Vol. 39, 1994

62. Branson, T., Olafsson, G., Schlichtkrull, H.: Huygens' Principle in Riemannian symmetric spaces. Math. Ann., V. 301, 1995, pp. 445-462

63. Branson, T., Olafsson, G.: Helmgoltz operator and symmetric spaces duality. Preprint, June 1995 